\title{
La «drôle de crise» de la CFE-CGC
}

Hésitations stratégiques et distorsions organisationnelles d'un syndicalisme catégoriel

The "phoney crisis" of the CFE-CGC: strategic hesitations and organizational distortions of a managers' and executives' trade union

Élodie Béthoux, Guillaume Desage, Arnaud Mias et Jérôme Pélisse

\section{OpenEdition}

\section{Journals}

Édition électronique

URL : http://journals.openedition.org/travailemploi/5422

DOI : 10.4000/travailemploi.5422

ISSN : 1775-416X

Éditeur

DARES - Ministère du Travail

Édition imprimée

Date de publication : 15 novembre 2011

Pagination : $5-22$

ISBN : 0224-4365

ISSN : 0224-4365

\section{Référence électronique}

Élodie Béthoux, Guillaume Desage, Arnaud Mias et Jérôme Pélisse, «La «drôle de crise» de la CFECGC », Travail et Emploi [En ligne], 128 | octobre-décembre 2011, mis en ligne le 01 juillet 2014, consulté le 01 mai 2019. URL : http://journals.openedition.org/travailemploi/5422 ; DOI : 10.4000/ travailemploi.5422 


\title{
Travail et Emploi
}

128 (octobre-décembre 2011)

Varia

Élodie Béthoux, Guillaume Desage, Arnaud Mias et Jérôme Pélisse

La « drôle de crise » de la CFE-CGC : hésitations stratégiques et distorsions organisationnelles d'un syndicalisme catégoriel

\begin{abstract}
Avertissement
Le contenu de ce site relève de la législation française sur la propriété intellectuelle et est la propriété exclusive de l'éditeur.

Les œuvres figurant sur ce site peuvent être consultées et reproduites sur un support papier ou numérique sous réserve qu'elles soient strictement réservées à un usage soit personnel, soit scientifique ou pédagogique excluant toute exploitation commerciale. La reproduction devra obligatoirement mentionner l'éditeur, le nom de la revue, l'auteur et la référence du document.

Toute autre reproduction est interdite sauf accord préalable de l'éditeur, en dehors des cas prévus par la législation en vigueur en France.
\end{abstract}

\section{revues.org}

Revues.org est un portail de revues en sciences humaines et sociales développé par le Cléo, Centre pour l'édition électronique ouverte (CNRS, EHESS, UP, UAPV).

\begin{abstract}
Référence électronique
Élodie Béthoux, Guillaume Desage, Arnaud Mias et Jérôme Pélisse, « La «drôle de crise » de la CFE-CGC : hésitations stratégiques et distorsions organisationnelles d'un syndicalisme catégoriel », Travail et Emploi [En ligne], 128 | octobre-décembre 2011, mis en ligne le 12 septembre 2012, consulté le 13 septembre 2012. URL : http://travailemploi.revues.org/5422
\end{abstract}

\author{
Éditeur : La documentation française \\ http://travailemploi.revues.org \\ http://www.revues.org \\ Document accessible en ligne sur : http://travailemploi.revues.org/5422 \\ Ce document est le fac-similé de l'édition papier. \\ Cet article a été téléchargé sur le portail Cairn (http://www.cairn.info)
}

\section{CAIRN}

Chercher, Repérer. Avancer.

Distribution électronique Cairn pour La documentation française et pour Revues.org (Centre pour l'édition électronique ouverte)

(c) La documentation française 


\title{
La « drôle de crise » de la CFE-CGC : hésitations stratégiques et distorsions organisationnelles d'un syndicalisme catégoriel
}

\author{
Élodie Béthoux ${ }^{(*)}$, Guillaume Desage ${ }^{(* *)}$, Arnaud Mias(**), Jérôme Pélisse ${ }^{(* * *)}$
}

\begin{abstract}
Retraçant les évolutions de la CFE-CGC depuis une quinzaine d'années et fondé sur une enquête articulant données quantitatives sur les participants au congrès de 2010 de la confédération, observations dans diverses structures et entretiens auprès de militants, l'article interroge les raisons des hésitations stratégiques répétées des dirigeants de cette organisation pour faire face aux problèmes qu'elle rencontre. Cette "drôle de crise» reflète une difficulté croissante à structurer une représentation homogène d'un groupe social, les cadres et encadrants, par nature hétérogène. L'article montre comment les transformations sociodémographiques de l'encadrement, qui ne se réduisent pas à une banalisation du groupe cadres, exercent des effets contrastés sur les structures organisationnelles et induisent des perceptions contradictoires des évolutions du contexte, dont l'acuité problématique pour la CFE-CGC se renforce avec la mise en auvre progressive de la loi d'août 2008 sur la représentativité syndicale. L'incapacité à dépasser ces clivages internes s'expliquerait par le maintien d'un syndicalisme particulièrement centré sur la section d'entreprise, confinant à un corporatisme d'entreprise dans les bastions syndicaux, et alimentant une forme de pléistocratie interne qui limite la portée du rôle des structures confédérales et interprofessionnelles.
\end{abstract}

La Confédération française de l'encadrement Confédération générale des cadres fait partie, avec la CFTC (Confédération française des travailleurs chrétiens), de ces or ganisations syndicales françaises dont on pronostique régulièrement le déclin irrémédiable, voire la disparition prochaine, ou dont on interroge au moins les conditions de la survie, et ce avant même les bouleversements qu'introduit la loi adoptée en août 2008 modifiant les règles de la représentativité syndicale. Jean-Marie PERNOT affirmait ainsi en 2005 : «la CFE-CGC semble avoir bel et bien parcouru son cyclehistorique» (p. 249). Une étude menée en 2010 et 2011 sur ce que signifie militer dans cette confédération (voir infra, encadré 1) permet d'aller au-delà de ces observations, qui restent trop rarement fondées sur des enquêtes sociologiques, alors même que les rôles de ces «petits syndicats» se révèlent parfois majeurs dans l'économie des relations professionnelles fran çaises. Il n'en reste pas moins que c'est une « drôle de crise» que traverse, depuis une quinzaine d'années, cette organisation singulière.

\footnotetext{
(*) MCF ENS Cachan, IDHE ; elodie.bethoux@idhe.enscachan.fr

(**) Ingénieur d'études

(***) MCF université de Rouen, DySoLa -IDHE Cachan; mias@ens-cachan.fr

$(* * * *)$ MCF université Versailles-Saint-Quentin-en-Yvelines, PRINTEMPS; jerome.pelisse@uvsq.fr
}

La CFE-CGC fut en effet longtemps considérée comme l'organisation syndicale phare et le princi pal porte-parole d'un groupe social en formation - le groupe cadres - qu'elle a, dans une conjoncture historique spécifique, contribué à structurer et stabiliser autour d'un système de représentations et de valeurs homogène. Elle a ainsi participé et soutenu le «travail social de définition et de délimitation qui a accompagné la formation du groupe et qui a contri bué en l'objectivant, à le faire être sur le mode du cela-va-de-soi » (Boltanski, 1982, p. 52). Dans les années 1980, la CFE-CGC, largement hégémonique au sein du syndicalisme des cadres et ingénieurs ${ }^{(1)}$, pouvait être appréhendée comme le modèle d'un syndicalisme catégoriel « cadres » et corporatiste jouant d'une dif férenciation constante avec les autres confédérations : «le groupe-cadres, par sa nature, son statut, sa relationaux autres catégories et par l'individualisme souvent affirmé de ses agents, reste particulièrement sensible à l'expression de sa propre identité, de sa spécificité, des attentes et revendications qui le définissent socialement. [...] Or la CGC, par son attachement à l'idéologie des classes moyennes, la pratique sociale qui la caracté-

(1) La CGC syndicalise alors $12 \%$ des cadres, loin devant l'UCC-CFDT (Union confédérale des cadres-CFDT; $3 \%$ ) et l'UGICT-CGT (Union générale des ingénieurs, cadres et tech niciens-CGT; 2,6 \%) (GrunberG, Mouriaux, 1979).

Travail et Emploi $n^{\circ} 128 \cdot 5 \bullet$ 
rise et la base professionnelle qui la compose, reste particulièrement bien adaptée à cette sensibilité corporatiste du groupe»(Groux, 1983a, p. 311). Cette «idéologie de la dif férenciation» conduit la CGC à construire sa stratégie revendicative par contraste avec les confédérations ouvrières dans le système français de relation $\mathrm{S}$ professionnelles ${ }^{(2)}$. Dans ces mêmes années 1980, la division crois sante du syndicalisme des cadres est perçue comme une réponse à l'hétérogénéité du groupe permettant de traduire les représentation s diverses qui y sont présentes (Groux, 1990). Derrière cette affirmation pointe l'idée que le travail symbolique et politique de représentation du groupe cadres - par lequel les organisations syndicales de cadres, pour assurer leur cohésion interne, produisent des représentations et des discours susceptibles de s'adresser à des caté gories et des cultures de métier fortement distinctes - rencontre des difficultés croissantes à s'unifier, dans une situation où les cadres se pensent de plus en plus comme partie du salariat (Groux, 1983b).

Les évolutions récentes confirment cette évolution. La hausse du nombre de cadres a pendant un temps (les Trente Glorieuses) conféré aux or ganisations syndicales de cadres " une assise sociale et une légitimité renforcée et durable» (Groux, 1983a, p. 315). Mais, en se prolongeant dans la période récente (le nombre de cadres en France a été multiplié par deux entre 1982 et 2002), elle aurait inversé ses effets conduisant à une «banalisation» du groupe cadres (B OufFartigue, 2001). Ces derniers partageraient les mêmes orientations sociopolitiques et les mêmes valeurs que les professions intermédiaires, indice de leur intégration dans une vaste classe moyenne. La thèse de la banalisation reste pourtant abondamment discutée aujourd'hui (Bensoussan, 2010). Thomas Amossé (2004, avec Delteil, 2011) pointe par exemple, aux côtés des facteurs de banalisation (sexe, âge, rémunération), des facteurs de dif férenciation (diplôme, durée du travail et stabilité de l'emploi), pour avancer $a$ contrario la thèse d'un maintien, voire d'un renforcement, de la frontière entre cadres et non -cadres, serait-ce sous des formes renouvelées.

Face à ces évolutions, qu'en est-il aujourd'hui de la stratégie de «différenciation» qui a longtemps caractérisé l'organisation? Comment les évolutions du groupe cadres affectent-elles le devenir de la CFE-CGC? Ces transformations du référent identitaire et de la base sociologique de la CFE -CGC sont ef fectivement au cœur des réflexions stratégiques des dirigeants de la confédération depuis quinze ans, à la suite notam ment du traumatisme des élections prud'homales de 1997, lors desquelles leur or ganisation passe derrière la CFDT dans le collège « encadrement» (à 21,9\% contre 41,4 \% en $1982^{(3)}$ ), entraînant le constat brutal d'une perte d'hégémonie dans la représentation syndicale des cadres. Dix années plus tard, l'adoption en août 2008 de la loi sur la représentativité syndicale, malgré une exception catégorielle arrachée au dernier moment, relance une situation de crise, suscitant ou réveillant des tensions, oppositions et déchirements internes au sein de la CFE-CGC.

Ces débats et tensions actuels sont-ils le symptôme d'une crise plus profonde ? Marquent-ils la longue agonie d'une organisation dont la base sociologique s'est effondrée depuis les années 1980 (voire les années 1960, lorsque sont apparues les unions spécifiques de cadres au sein des centrales « ouvrières»), mais qui reste sous perfusion de sa reconnaissance institution nelle, tant que l'exception catégorielle est préservée ? La crise que traverse la CFE -CGC apparaitrait alors comme la panne d'une stratégie de développement et d'implantation dans le système français de rela tions professionnelles, jusque -là fondée sur une présomption irréfragable de représentativité(4) et sur une capacité à porter le discours de la dif férenciation. Ces deux piliers seraient mis à mal par les évolutions diverses qui affectent aujourd'hui son environnement : évolutions institutionnelles, avec la mise en œuvre de la loi du 20 août 2008; stratégiques, avec l'évolution des positions de l'État et des or ganisations patronales à son égard; et morphologiques avec les transforma tions sociodémographiques du groupe cadres.

L'article analyse comment ces transformations sont appréhendées par les responsables de la CFE -CGC pour comprendre leurs hésitations stratégiques récurrentes. Il s'inscrit dans la perspective des travaux engagés depuis une dizaine d'années, principalement dans le monde anglosaxon, sur les stratégies syndicales face à la crise et au déclin, souvent considérées sous l'angle de la «revitalisation», et qui prennent la forme notamment de campagnes de syndicalisation, d'action politique, de construction d'alliances, notamment avec les mouvements sociaux (TURNER et al., 2001; FREGE, KelLy, 2004). Ainsi, dans leur comparaison interna tionale, Carola M. F REGE et John K ELLY proposent un modèle explicatif des choix stratégiques des syndicats qui fait intervenir trois variables contextuelles - les

(3) Dans le même temps, la CFDT est passée de 17,5\% à $31,5 \%$. Paul B ouffartigue (2001) interprète d'ailleurs cet événement comme un signe de la montée d'une conscience salariale chez les cadres, c'est-à-dire d'un sentiment de rapprochement et de solidarité avec les autres salariés.

(4) Et, avec elle, une reconnaissance et une participation institutionnelles, notamment à travers un paritarisme catégoriel dans la gestion de caisses spécifiques de retraites et d'allocations chômage.

(2) Aujourd'hui encore, la singularité de la CFE -CGC le paysage syndical français apparaît très nettement. s'affiche comme un syndicat réformiste, très plutôt peu enclin à s'engager dans les conflits collectifs. Mêm si l'organisation revendique son indépendance politique, ses militants et sympathisants votent nettement plus à droite que ceux des autres or ganisations syndicales. D'après un sondage Liaisons sociales-Louis Harris du 20 mars 2011, $70 \%$ des sondés se déclarant proches de la CFE -CGC auraient voté à droite ou à l'extrême droite aux dernières élections cantonales. 
stratégies de l'État et des employeurs, leschangements économiques et sociaux et le contexte institutionnel des relations professionnelles - dont l'influence est médiatisée par les structures syndicales et par des processus de « cadrage» qui renvoient aux identités syndicales et aux répertoires d'action disponibles (5). Il s'agit de comprendre comment les évolutions du contexte de l'organisation sont traduites par le biais des structures syndicales et celui des processus de cadrage dont elles sont le théâtre et qui contribuent à constituer la réalité (voir G IRAUD, 2006, pour une perspective proche portant sur les reconfigurations des rapports des confédérations ouvrières à la grève). En retraçant les tensions et of fres stratégiques distinctes, et parfois opposées, qui structurent les débats au sein de la CFE-CGC, en proposant une sociographie de ses dirigeants et en analysant les clivages or ganisationnels qui la traversent, c'est ce travail cognitif que nous étudions ici.

Nous montrons ainsi que la «drôle de crise» que traverse la confédération, et ses hésitations stratégiques répétées depuis quinze ans, s'expliquent par une difficulté croissante de l'organisation à structurer une représentation homogène d'un groupe social par nature hétérogène ${ }^{(6)}$. Après avoir pointé les manifestations contrastées de cette « drôle de crise» (partie 1), nous analysons la manièredont les militants et responsables syndicaux perçoivent et réagissent à ces évolutions (partie 2). Comprendre les débats et décisions des dirigeants de ce syndicat nécessite alors de montrer comment les transformations sociodémographiques des encadrants exercent des effets contrastés sur les structures organisationnelles du syndicat et alimententdes distorsions dans la manière de définir le groupe social que ce dernier entend représenter (partie 3). Nous formulons enfin l'hypothèse que les difficultés de l'organisation à faire fi de ses clivages internes s'expliquent essentiellement par le maintien d'un syndicalisme centré sur la section d'entreprise, articulantun ensemble de pratiques et de représentatio ns syndicales héritées des traditions du syndicalisme catégoriel qui, si elles sont pour partie façonnées par les caractéristiques du système français de relations professionnelles, sont particulièrement accentuées dans le cas de la CFE-CGC (partie 4).

(5) L'influence des travaux sur les mouvements sociaux, notamment ceux de D. S Now (Hunt, Benford, Snow, 1994) et de C. Tilly (McAdam, Tarrow, Tilly, 2001), est explicite.

(6) Il ne s'agit pas d'affirmer ainsi le caractère radicalement nouveau de cette hétérogénéité. Le syndicalisme des cadres s'est toujours construit en faisant face à l'hétérogénéité du groupe social dont il s'est fait le porte-parole. Toutefois, les circonstances historiques de coconstruction du syndicalisme cadres et de la catégorie cadres décrites par Luc B OLTANSKI (1982) - qui ont permis ce caractè re inédit d'une catégorie à la composition hétérogène dont l'existence est en même temps indéniable pour ses propres membres et pour ceux des autres groupes - semblent aujourd'hui passées.

\section{Encadré 1 \\ Du congrès aux militants, méthodologies et données d'enquête}

Initiée lors du $34^{\mathrm{e}}$ congrès de la CFE-CGC qui s'est tenu à Reims en février 2010, l'étude débute par une invitation faite par la confédération à J. Pélisse à participer à ce congrès comme "expert» lors de deux tables rondes ${ }^{(7)}$. Les auteurs proposent alors de mener une étude sur l'identité et les pratiques des responsables syndicaux présents lors du congrès. Plusieurs matériaux empiriques sont ainsi produits : observations conduites lors de ce congrès de la CFE-CGC et lors d'ateliers, colloques et séminaires organisés par ses instances territoriales (UL [Unions locales], UD [Unions départementales], UR [Unions régionales] ) courant 2010 et 2011; questionnaires remplis par les congressistes présents à Reims ( $n=310$ sur environ 500 participants); entretiens approfondis auprès de militants et responsables syndicaux menés à tous les niveaux de l'organisation en 2010 et 2011 en région parisienne, dans le Nord et en Rhône-Alpes notamment ( $n=61)$.

Observer et participer aux enjeux du congrès de 2010 - placé sous le slogan «Notre histoire a de l'avenir » - constituent une occasion privilégiée pour interroger les hésitations stratégiques et les débats de l'organisation qui sont au cœur de notre analyse. De même, recueillir des données sur les congressistes aussi bien en termes sociodémographiques que sur leurs perceptions et opinions quant à la loi de 2008, le rapprochement possible ou non avec d'autres syndicats, ou leur attachement au catégoriel - ouvre la possibilité de dépasser les sources classiques constituées par la presse syndicale et d'appréhender les profils sociodémographiques des principaux dirigeants de l'organisation. Enfin, les observations et entretiens approfondis que nous avons menés ont permis de préciser, mais aussi complexifier l'analyse du travail de construction identitaire et de distorsions organisationnelles au cœur de notre étude.

Pour autant, notre entrée n'est pas sans biais : moins parce que nous étions financés par la confédération, qui a entériné toutes nos propositions de questionnaires, choix de secteurs à étudier ou personnes à rencontrer; mais plutôt parce que les militants rencontrés et questionnés - très majoritairement parmi les congressistes présents à Reims - ne sont pas représentatifs de l'ensemble des adhérents, délégués ou militants de la CFE-CGC.

La population enquêtée est en effet avant tout construite par les règles et pratiques de désignation des représentants par les fédérations, souvent variables d'une structure à l'autre. Les propriétés

(7) On peut s'interroger sur ce choix, Jérôme Pélisse n'étant pas un spécialiste du syndicalisme, ni du syndicalisme-cadres. Sa fonction - il est alors maître de conférences à l'université de Reims (lieu du congrès) - comme ses travaux sur les 35 heures ou la conflictualité au travail pourraient l'expliquer. Mais la chargée de communication qui l'a invité met en avant des raisons médiatiques (contribution et interviews dans des journaux, radios ou télévisions ) qui montrent sans doute l'importance de la communication dans les stratégies prises au niveau de la direction confédérale. 
militantes et sociodémographiques des participants dépendent de ce qui se joue lors du processus de mandatement : des représentants peuvent par exemple être désignés parce qu'ils sont plus légitimes (en raison de leur ancienneté, de leurs prises de position, de leur fonction, etc.) ou plus disponibles (par exemple parce qu'ils sont retraités) que d'autres. Le nombre d'invitations envoyées aux fédérations dépend, quant à lui, en principe, de la part d'adhérents que chacune représente, sachant que toutes n'utilisent pas le nombre de "sièges" auquel elles ont droit. Comme le soulignent Cécile Guiltaume et Sophie PocHIc dans leur étude du syndicalismecadres à la CFDT, «malgré les biais que présente une telle enquête (militants en responsabilité envoyés par leur fédération ou syndicat, dont le profil est souvent distinct de celui des simples adhérents), elle est une source classique d'informations sur le profil des militants " $\left(2009\right.$, p. 537) ${ }^{(8)}$. De fait, ce sont même ces biais entre répondants, militants et adhérents qui fondent ici l'intérêt de ces données, sachant que ces responsables syndicaux ont des attributs essentiels et communs : le fait d'être mandaté par au moins une composante de la CFE-CGC (confédération, fédération, syndicat, UD, UR), mais aussi de se trouver au même endroit, au même moment, dans un cadre défini par les enjeux et le contexte du congrès, autant d'éléments à prendre en considération pour interpréter leurs réponses.

\section{La "drôle de crise" de la CFE-CGC : 1997-2012}

Comme la drôle de guerre, cette «drôle de crise» tord les représentations habituelles du phénomène, en conjuguant symptômes de déclinet résultats électoraux en progression. On y trouve même une ligne Maginot, qui correspond ici à l'exception catégorielle contenue dans la loi d'août 2008 et autour de laquelle s'affrontent partisans du mouvement (vers une confédération généraliste) et défenseurs des acquis (catégoriels).

\section{Les symptômes d'un déclin...}

La crise que rencontre la CFE-CGC se manifeste d'abord par une baisse régulière du nombre d'adhérents. Jean-Michel Denis (2005) rappelle ainsi que leurs effectifs sont passés de 282650 en 1984 à 186397 en 1999, selon les données fournies par la confédération. Lors de son congrès de 2010, elle n'en dénombre même plus que 113000 dans son rapport d'activité.

(8) Une journée d'étude organisée le 26 mars 2012 par le réseau thématique «relations professionnelles » de l'Association française de sociologie a visé justement à travailler cette forme et ce moment social singulier qu'est un congrès syndical, et ce qu'il nous apprend sur les or ganisations et les militants qui les font vivre.
La baisse du nombre d'adhérents s'inscrit dans la tendance générale à la désyndicalisationobservée en France depuis la fin des années 1970. Mais l'érosion paraît se prolonger pour la CFE-CGC, alors qu'on observe, depuis le milieu des années 1990, une stabilisation de la proportion de syndiqués en France (Amossé, 2004). Surtout, cette baisse s'opère dans un contexte plutôt favorable au syndicalisme des cadres, marqué par le maintien voire la poussée d'un rapport plus ouvert de ces derniers à l'égard du mouvement syndical. "Selon les enquêtes du Cevipof, la confiance que l'on porte aux syndicats a bien plus augmenté chez les cadres que chez les membres des autres catégories entre 1978 et 1997 : on passe de $34 \%$ à $62 \%$ chez les cadres du privé et de $46 \%$ à $67 \%$ chez les cadres du public», soulignent ainsi GuILlaume et Pochic (2009, p. 535, citant Rouban, 2001). Ces évolutions du rapport des cadres au syndicalisme se sont traduites par une stabilité de leur taux de syndicalisation depuis plus de vingt ans. En 2003, une enquête aboutissait à un taux s'établissant à $14,5 \%$, soit presque deux fois plus que la moyenne (A mossé, 2004) ${ }^{(9)}$. Rappelons qu'à la fin des années 1970, le taux de syndicalisation des cadres s'établissait autour de $20 \%$, ce qui était alors évalué comme un syndicalisme «minoritaire» (Grunberg, Mouriaux, 1979). Autrement dit, la catégorie cadres semble avoir relativement mieux résisté que les autres à la chute de la syndicalisation dans les années 1980. Il faut toutefois relativiser cette santé du syndicalisme-cadres, car une dif férence très nette se manifeste entre cadres du public et cadres du privé : $25 \%$ des ingénieurs et cadres de la fonction publique déclarentêtre membres d'un groupe syndical ou professionnel, contre seulement $7,5 \%$ des cadres d'entreprises. La CFE-CGC, relativement peu implantée dans la fonction publique, a donc moins bénéficié que les autres confédérations syndicales de ce maintien du syndicalisme-cadres dans le public.

La confédération ne bénéficie par ailleurs que d'une faible aura médiatique. Son taux de notoriété, tel que des sondeurs sollicités par la direction confédérale lors de la préparation du congrès de Reims en février 2010 le font apparaître, se situe clairement en deçà de celui des autres centrales syndicales : seuls $27 \%$ des Français interviewésà cetteoccasion disent «connaitre, ne serait-ce que de nom», la CFE-CGC, contre $38 \%$ pour la CFTC et $53 \%$ à $57 \%$ pour FO, la CFDT ou la CGT ${ }^{(10)}$. Son président, Bernard Van Craeynest, est peu connu (voir encadré 2) et

(9) Ce taux doit toutefois être interprété avec prudence car il correspond à la proportion de cadres déclarant adhérer à un groupement syndical ou professionnel, ce qui est une nuance importante pour les cadres et conduit sans doute à une certaine surestimation. Pour une approche étudiant simultanément les engagements syndical et associatif des cadres, voir Bensoussan (2010).

(10) Sondage OpinionWay pour la CFE-CGC, source interne, janvier 2010 . 
moins médiatisé que les leaders d'autres centrales syndicales. Un nombre non négligeable de militants de la CFE-CGC considèrent la faible notoriété de l'organisation comme un handicap de poids pour l'exercice de leur mandat, les pénalisant en particulier au moment des élect ions professionnelles. La communication constitue pourtant un service central au sein de la confédération, qui a multiplié ces dernières années l'usage des nouvelles technologies (site Web, compte Facebook, sondages en ligne, etc.).

\section{Encadré 2
Bernard Van Craeynest, un responsable
syndical peu connu}

Né en 1957, Bernard Van Craeynest rejoint l'établissement de la Snecma à Évry-Corbeil en 1979, suite à l'obtention d'un BTS physico-métallographe. Après deux années au service de sous-traitants, il y est embauché comme technicien supérieur au laboratoire de la direction technique. En 1989, il devient assistant ingénieur. En 1995-1996, il est promu cadre et est rattaché aujourd'hui à la direction de la qualité.

Adhérant rapidement à la CFE-CGC, il devient délégué syndical central en 1983 et accède à un mandat de représentant au CE de Corbeil-Essonnes en 1987. À partir de 1988, il participe à l'animation de la Fédération de la métallurgie, comme membre d'abord du conseil national, puis du comité directeur en 1992, avant d'être élu vice-président du bureau en 1995, puis secrétaire national, chargé de l'économie et de la politique industrielle en 1998, vice-président délégué en 2001 , et enfin président de la Fédération en 2004. En parallèle, il accède à différents mandats au sein du Syndicat national des cadres et techniciens de l'aéronautique et de l'astronautique (SNCTAA), devenu aujourd'hui le syndicat Aéronautique, espace, défense (AED), partie constitutive de la Fédération de la métallurgie qui en concentre un tiers des adhérents.

Bernard Van Craeynest siège au comité confédéral de la CFE-CGC depuis 1992. II devient membre titulaire du comité directeur confédéral en 2004, est élu président de la CFE-CGC en décembre 2005, à la suite du décès du président en place, Jean-Luc Cazettes, puis réélu en décembre 2006 et en février 2010.

Les événements qui corroborent l'idée d'un déclin de l'organisation ces quinze dernières années ne manquent pas. Nous avons déjà évoqué le trau matisme des élections prud'homales de 1997. Récemment encore, en janvier 2012, la perte de la codirection de l'Agirc (Association générale des institutions de retraite des cadres) au profit de la CFDT, si elle était attendue, n'en constitue pas moins un événement pour cette institution symbole du paritarisme catégoriel de la CFE-CGC qu'elle avait présidée en alternance avec le CNPF (Conseil national du patronat français), puis avec le Medef, depuis sa création en 1947, et qui avait contribué à l'inscription durable de la catégorie cadres dans le monde social (11). Fin 2011, la CFE-CGC perdait déjà la présidence de la Caissenationale d'assurance vieillesse, qu'elle occupait depuis dix ans, au profit de Force ouvrière ${ }^{(12)}$. C'est ainsi la première fois de son histoire que la CFE -CGC n'exerce pas de mandat à la tête des institutions-clés du paritarisme.

\section{... relativisés par des résultats électoraux en progression}

Si les symptômes d'un déclin sont clairement perçus par les responsables de la CFE -CGC, la dernière décennie a aussi donné lieu à des évolutions plus encourageantes dans les résultats de l'organisation aux élections professionnelles et prud'homales, contribuant à créer ce clima $t$ de " drôle de crise " que nous avons perçu dans nos échanges avec les dirigeants et les militants de la confédération. Ainsi, lors des deux élections prud'homales qui ont suivi la « débâcle» de 1997, la CFE-CGC s'est maintenue à un niveau équivalent à celui de l'élection précédente, enrayant la chute continue de ses proportions de voix obtenues depuis 1982, avant de redevenir en 2008 la première organisation syndicale dans le collège cadre, progressant même auprès de l'ensemble des salariés (voir tableau 1).

Tableau 1 : Résultats aux élections prud'homales, collège «encadrement» 1979-2008 (pourcentage des suffrages exprimés)

\begin{tabular}{|l|c|c|c|c|c|c|c|}
\hline & $\mathbf{1 9 7 9}$ & $\mathbf{1 9 8 2}$ & $\mathbf{1 9 8 7}$ & $\mathbf{1 9 9 2}$ & $\mathbf{1 9 9 7}$ & $\mathbf{2 0 0 2}$ & $\mathbf{2 0 0 8}$ \\
\hline CFE-CGC & 36 & 41,4 & 29,5 & 27,2 & 21,9 & 22,8 & 27,8 \\
\hline CFDT & 17,8 & 17,5 & 21,3 & 23,5 & 31,5 & 28,6 & 22,9 \\
\hline CGT & 17 & 13 & 14,6 & 14 & 16,2 & 15,8 & 16,9 \\
\hline
\end{tabular}

Sources : Bouffartigue, 2001, d'après ministère du Travail pour la période 1979-1997; Le Monde pour 2002; ministère du Travail, de l'Emploi et de la Santé pour 2008.

La CFE-CGC renforce égal ement sa présence dans les entreprises. D'après l'enquête REPONSE, la part d'établ issements disposant d'au moins un délégué CFE-CGC est passée de 13,8\% à 15,6\% entre 1992 et 2004. La progression se manifeste quelle que soit la taille de l'établissement, même si elle est plus prononcée dans les grands établis sements (Pernot, Pignoni, 2008, p. 145). En 2006,

(11) Ce désaveu de la CFE-CGC, vers laquelle les voix patronales au sein du conseil d'administration ne se sont pas majoritairement portées, était attendu dans la mesure où la CFE-CGC avait refusé de signer l'accord Agirc-Arrco de mars 2011. Sur les raisons, voir J. -L. BESNARD, «L'accord sur les retraites complémentaires Agirc-Arrco du 18 mars 2011 : "accord historique" ou simple "compromis conservatoire" "), Revue de l'Ires, no 69, 2011/2, pp. 69-110.

(12) Dans ce cas, Danièle Karniewicz ne pouvait se représen ter pour un troisième mandat. Mais la victoire de FO reflète l'évolution du rapport de force syndical, suite à l'accord AgircArrco de mars 2011 ou à l'accord sur le paritarisme en cours de signature fin février 2012, deux accords que la CFE-CGC, avec la CGT, a (ou devrait) refusé(er) de signer, contrairement à la CFDT, la CFTC et FO. 
la CFE-CGC recensait 22500 représentants dans les instances sociales et économiques et 7500 délégués syndicaux, soit 30000 adhérents chargés de diverses fonctions institutionnelles, chiffres qu'elle confirme dans son rapport d'activité au congrès de février 2010. Cette présence accrue dans les entreprises s'accompagne d'un maintien, voire d'une légère progression, aux élections aux comités d'entreprise. Tous collèges confondus, les résultats de la CFE-CGC sont en légère progression depuis le début des années 2000 (5,8 \% en 2001, 6,3\% en 2003, 6,5\% en 2005) et, dans le collège« ingénieurs et cadres », la CFE-CGC confirme sa première place (voir tableau 2).

Tableau 2 : Résultats aux élections aux comités d'entreprise 1997-2006 (pourcentage des suffrages exprimés)

\begin{tabular}{|l|c|c|c|c|c|}
\hline & $\mathbf{1 9 9 7 -}$ & $\mathbf{1 9 9 9 -}$ & $\mathbf{2 0 0 1 -}$ & $\mathbf{2 0 0 3 -}$ & $\mathbf{2 0 0 5 -}$ \\
& $\mathbf{1 9 9 8}$ & $\mathbf{2 0 0 0}$ & $\mathbf{2 0 0 2}$ & $\mathbf{2 0 0 4}$ & $\mathbf{2 0 0 6}$ \\
\hline \multicolumn{7}{|c|}{ Troisième collège } \\
\hline CFE-CGC & 26,7 & 24,7 & 24,5 & 26 & 25,6 \\
\hline CFDT & 18,8 & 21,1 & 20,4 & 19,4 & 19,4 \\
\hline \multicolumn{7}{|c|}{ Deuxième collège } \\
\hline CFDT & 21,3 & 22,6 & 22,1 & 20,6 & 19,9 \\
\hline CGT & 15,1 & 15,7 & 16,7 & 17,4 & 17,9 \\
\hline CFE-CGC & 12,4 & 12 & 12 & 11,5 & 10,9 \\
\hline CGT-FO & 10,7 & 11,7 & 11,9 & 11,8 & 11,5 \\
\hline
\end{tabular}

Source : JACOD, 2008a et 2008b.

À la fin des années 2000, la situation est donc contrastée, au sein d'un paysage syndical lui-même en plein bouleversement, marqué par une position commune inédite de la CGT, la CFDT, le Medef et la CGPME en avril 2008, reprise dans une loi votée en août la même année. La réforme de la repré sentativité fondant la capacité des organisations syndicales à pouvoir négocier et signer des accords (et bénéficier de moyens, notamment en heures de délégation syndicale) constitue en ef fet un choc particulièrement brutal pour tous les «petits» syndicats et notamment la CFE-CGC.

\section{Une ligne Maginot? L'exception catégorielle dans la loi du 20 août 2008}

La loi du 20 août 2008 «portant rénovation de la démocratie sociale et réforme du temps de travail» a en effet introduit le principe d'une mesure régulière de la représentativité des syndicats de salariés en fonction de leurs résultats aux élections professionnelles, rompant avec le principe de la présomption irréfragable de représentativi té héritée de l'après guerre. À l'horizon 2013, première échéance fixée par la loi, il faut s'attendre à des évolutions importantes dans les rapports entre or ganisations syndicales, entre équipes syndicales et adhérents, et entre sections syndicales et salariés-électeurs.

La loi de 2008 soulève, pour la CFE-CGC, d'importants enjeux identitaires qui mettent en débat tant l'autonomie et l'indépendance du syndicat vis-à-vis d'autres organisations syndicales que sa spécificité catégorielle. Pour la CFE-CGC, la loi d'août 2008 apparaît en ef fet lourde d'enjeux, en ce qu'elle adopte une logique électorale qui, sans l'existence d'une exception catégorielle spécifique à cette confédération (voir encadré 3), est suscep tible de la faire disparaître à terme.

\section{Encadré 3 \\ L'exception catégorielle dans la loi d'août 2008}

L'article L. 2122-1 du Code du travail révisé en 2008 impose désormais aux organisations syndicales de recueillir "au moins $10 \%$ des suffrages exprimés au $1^{\text {er }}$ tour des élections professionnelles du comité d'entreprise, de la délégation unique du personnel ou, à défaut, des délégués du personnel " pour être jugées représentatives et participer aux négociations. Toutefois, une exception a été mise en place par la loi du 20 août 2008, codifiée à l'article L. 2122-2, au bénéfice des syndicats affiliés à une organisation nationale catégorielle.

À ce titre, le Code du travail prévoit que «dans l'entreprise ou l'établissement, sont représentatives, à l'égard des personnels relevant des collèges électoraux dans lesquels leurs règles statutaires leur donnent vocation à présenter des candidats, les organisations syndicales catégorielles affiliées à une confédération syndicale catégorielle interprofessionnelle nationale qui satisfont aux critères de l'article L. 2121-1 et qui ont recueilli au moins $10 \%$ des suffrages exprimés au premier tour des dernières élections des titulaires au comité d'entreprise ou de la délégation unique du personnel ou, à défaut, des délégués du personnel dans ces collèges, quel que soit le nombre de votants ". En d'autres termes, alors que les syndicats généralistes doivent obtenir $10 \%$ des suffrages obtenus sur l'ensemble des collèges pour être jugés représentatifs, le pourcentage obtenu par un syndicat catégoriel ne sera calculé que sur le ou les collèges qu'il représente.

Cependant, cette exception catégorielle, que prévoyait déjà, à la suite de l'insistance dupatronat, la position commune d'avril 2008, apparaît fragile : «ce qu'une loi fait, une autre loi peut le défaire», expliquent plusieurs des responsables et militants rencontrés. C'est pourquoi elle constitue peut -être une ligne Maginot dans ce contexte de « drôle de crise» que nous décrivons ici.

De fait, malgré cette protection, la position de la confédération a toujours été de dénoncer vigoureu sement cette réforme, perçue par la CFE-CGC mais aussi par la CFTC, FO ou l'UNSA (Union nationale des syndicats autonomes) comme une volonté de domination des deux principaux syndicats, signa taires de la position commune. Le principe même de cette exception est par ailleu rs critiqué et contesté, y compris devant les tribunaux : la CFTC et FO ont ainsi rapidement engagé des recours contre cette disposition, créant une situation d'incertitude 
juridique, au niveau jurisprudentiel, qui s'ajoute à l'incertitude légale. Ces affaires trouvent des débouchés après le congrès de Reims qui se tienten février 2010. Bien qu'elles se soldent finalement par une réaffirmation de l'exception catégorielle ${ }^{(13)}$, elles témoignent d'attaques répétées qui font craindre que celle-ci ne saute et qui pèsent dès lors sur les stratégies des dirigeants de la confédération et sur les pratiques des militants. Au final, le contexte des dernières années n'est pas sans danger pour la confédération : si elle n'est pas dénuée d'atouts et si on ne peut, selon nous, considérer que la CFE-CGC a parcouru son cycle historique, il est clair que la «drôle de crise » qu'elle traverse depuis une quin zaine d'années n'est pas sans conséquence sur son fonctionnement interne. Au-delà de ces signes extérieurs, la crise se manifeste aussi par des épisodes récurrents de tensions internes, voire de conflit ouvert au sein du comité directeur de la CFE-CGC ou lors de ses congrès, traduisant des hésitations stratégiques fortes qui débouchent sur une grande difficulté et un certain immobilisme pour s'adapter aux nouvelles donnes institutionnelles et morphologiques qui marquent les années 2000.

\section{Des hésitations stratégiques qui traduisent un aggiornamento impossible?}

\section{Un renouvellement en trompe-l'œil? Des classes moyennes stressées...}

Ces hésitations stratégiques se manifestent sur différents plans. Elles se traduisent d'abord par un renouvellement du discours et de la plate -forme revendicative, qui se fonde en réalité pour une bonne part sur un retour aux thèmes traditionnels que portait la CFE -CGC(14). Ainsi est fortement remise en avant, à la fin des années 2000, la défense des classes moyennes, discours historiquement fondateur propre à la CFE -CGC dans le paysage du syndicalisme-cadres, même si c'est justement, comme l'a montré B OLTANSKI (1982, pp. 142-146

(13) Les deux principales décisions, défavorables à la CFECGC en première instance, ont donné lieu à des décisions de la Cour de cassation en avril 2010, annulant le jugement du tribunal de Brest mais sans se prononcer sur " l'inégalité de traitement » relevée par ce tribunal à propos de la CFE -CGC, et du Conseil constitutionnel en octobre 2010, suite à une question prioritaire de constitutionnalité sur l'article L. 2122-2 du Code du travail: le Conseil constitutionnel estime que le législateur n'a pas méconnu le principe de liberté syndicale et que l'exception catégorielle n'est pas contraire au principe d'égalité devant la loi.

(14) On peut noter toutefois l'émer gence, qui se traduit aussi par une série d'actions (brochures, platesformes revendicatives, négociations et signatures d'accords, etc.), des thèmes de l'égalité professionnelle entre hommes et femmes ou du handicap, ce dernier, notamment, singularisant la CFE-CGC par rapport aux autres confédérations. La thématique du logement, également investie de manière originale par la confédération, est quant à elle davantage liée à celle, plus ancienne, des classes moyennes. notamment), en s'émancipant de cette seule rhéto rique, si présente dans les mouvements d'ingénieurs et de cadres des années 1930, que le groupe « cadre et encadrement» que prétend représenter la CGC a pu prendre de la consistance.

De même, l'émer gence de la problématique du «stress» des cadres à la fin des années 1990, si elle s'inscrit dans un moment de durcissement des positions de la confédération à l'égard du gouverne ment et du patronat ${ }^{(15)}$, n'est pas sans ambivalence. D'un certain côté, l'investissement précoce de la CFE-CGC sur cette thématique (via en particu lier l'action d'un médecin du travail militant à la CFE-CGC, Bernard Salengro) et l'écho médiatique qu'il rencontre marquent un déplacement à l'égard de revendications plus traditionnellement centrées sur la gestion des entreprises et un « syndicalisme de (contre-) propositions industrielles» (GRoux, 1990). Corinne Delmas (2011) montre toutefois, par comparaison avec l'appropriation de ce thème par la CFDT Cadres, que l'investissement dans une réflexion sur l'organisation du travail en tant que telle est relati vement faible, et emprunte davantage au registre de la scandalisation visant avant tout à objectiver l'ampleur du phénomène. Elle souligne également le caractère catégoriel de cette mobilisation autour du thème du stress, là où la CFDT l'insère dans une réflexion plus large sur l'intensification du travail et relativise l'idée d'une " souffrance au travail » des cadres. Le thème du stress des cadres n'est -il pas, d'ailleurs, la reformulation d'une rhétorique beau coup plus ancienne, comme celle du « malaise des cadres» dont on trouve trace dans le discours de la CGC dès les années 1944-1947 et qui resurgit périodiquement depuis (BolTANSKI, 1982)? De fait, en en restant à une manière actualisée de dénoncer ce récur rent «malaise des cadres » (qu'illustre par exemple la focalisation sur le baromètre qu'elle a construit en matière de stress des cadres), la CFE-CGC ne semble pas réussir à capitaliser et à construire une véritable expertise sur cette thématique (sauf dans quelques entreprises phares comme France Télécom), ni à transformer médiatiquement, électoralement ou en forces militantes, son investissement sur ce thème.

\section{Participations intermittentes aux intersyndicales de 2009 et 2010 et fusion avortée : une continuité dans les volte-face?}

Les hésitations stratégiques de la CFE-CGC s'expriment également dans ses positions oscillantes à l'égard des initiatives gouvernementales, patronales et des autres confédérations. À la fin des années

(15) Lorsque survient le choc des prud'homales de 1997, la stratégie de la CFE-CGC oscille déjà depuis plusieurs années entre un positionnement réformiste traditionnel et une attitude contestataire (ChOfFat et Verrier, 2006). Le congrès de 1996, au lendemain du mouvement sur les retraites de l'hiver 1995, conduit Marc Vilbenoît, malmené, à durcir le ton à l'égard de ses interlocuteurs. 
1990, la posture se fait plus contestatairesur certains aspects - et notamment le temps de travail puisque, au moment de la réduction du temps de travail, la CFE-CGC relaye lar gement les revendications des cadres qui refusent d'être écartés du proces sus comme les employeurs l'envisagent, avant de contester fortement l'inventi on du forfait jour, y compris au niveau judiciaire et européen. Mais la CFE-CGC signe tout de même la réforme de l'assut rance chômage en 2000, donne son aval au projet de réforme sur les retraites en 2003 et se distingue des autres organisations syndicales en ne rejetant pas d'emblée le « contrat première embauche » en 2006. Pour autant, elle participe à l'intersyndicale unitaire inédite qui se met en place le 5 janvier 2009 ainsi qu'aux grandes manifestations et mouvements de grève interprofessionnels du printemps 2009 (BÉroud, Yon, 2012) et refuse de signer , pour la première fois de son histoire, un accord portant sur l'Agirc-Arrco en mars 2011 (voir supra).

La centrale catégorielle s'est aussi illustrée lors de la récente réforme des retraites : elle est impliquée au sein de l'intersyndicale qui refuse la réforme prévue par le gouvernement, y compris en participant aux premières grandes manifestations de l'automne 2010, mais son implication faiblit face à l'intransigeance du gouvernement qui rejette toute négociation - constat sans doute plus vrai au sommet que sur le terrain, où plusieurs sections CFE-CGC décident par exemple de manifester sans banderoles, même aux manifestations auxquelles n'appelle plus, ou dans lesquelles ne s'implique plus véritablement la confédération. L'essentiel pour les dirigeants de la CFE-CGC est en ef fet l'ouverture de ces négociations, tant l'enjeu-clé se joue en réalité ensuite pour les cadres lors de la renégociation des accords sur les retraites complémentaires, où le sens du compromis et de la négociation ne suffira pas pour signer l'accord qui en découlera. Or, la question des retraites touche particulièrement la CFE-CGC, car :

«C'est le refus d'être intégrés au régime général et par là, assimilés aux autres salariés, d'être des sala riés comme les autres, qui, à ses origines, assure la cohésion de la CGC. On ne comprendrait pas le rôle que joue, encore aujourd'hui, la défense du régime de retraite des cadres dans l'idé ologie de la CGC si on ne voyait, d'une part, que la résistance à la fusion dans le régime général a constit ué la principale occasion (avec la défense des avantages fiscaux et de la hiérarchie des salaires) de mobiliser dans une action commune, des catégories relativement hétérogènes et, d'autre part, que le rattachement à un même régime de retraite a constitué, et, d'une certaine façon, constitue encore aujourd'hui, le principal lien entre des agents et des groupes dispersés sous un très grand nombre de rapports. L'affiliation à une caisse de retraite de cadres est toujours le critère au nom duquel peut être revendi quée l'appartenance à la catégorie et, par là, au moyen duquel peut être construite la définition la plus extensive du groupe» (BOLTANSKI, 1982, p. 148).
C'est pourquoi la défaite du mouvement syndical, et plus spécialement de la CFE -CGC (qui se prolonge pour elle lors des négociations Agirc-Arrco), ébranle tout particulièrement la confédération. Cette phase d'hésitations pronon cées entre stratégies d'accompagnement et d'opposition face aux projets gouvernementaux ou patronaux manifeste encore une continuité dans l'histoire de l'organisation, marquée par des compromis et des «systèmes d'alliances [...] polymorphes», contrastés et évolutifs (G Roux, 1990, p. 138). Et après elle, on peut donc sans doute repérer dans les dernières années de la décennie 2000 une certaine autonomisation de la confédé ration à l'égard des propositions patronales, ainsi qu'une forme de normalisation de l'or ganisation par rapport aux autres confédérations. C'est alors la stratégie de dif férenciation et la position originale de la CFE-CGC dans le jeu des relations professionnelles qui tendent à s'affaiblir.

À cet égard, les tentatives de rapprochement et même de fusion avec l'UNSA constituent des symptômes encore plus explicites de ces hésitations stratégiques de la CFE -CGC et des interrogations que soulève son positionnement dans l'espacesyndical et des relations professionnelles. Il faut d'abord souligner qu'un rapprochement avec d'autres organisations syndicales avait été envisagé dès le début des années 1990 (C hoffat, Verrier, 2006). Cette réflexion sur une possible «redéfinition de l'offre syndicale» est ensuite précipitée par le traumatisme de 1997 : «l'autodissolution fut (même) un temps à l'ordre du jour, tout comme l'idée de rejoindre l'UNSA» (Pernot, Pignoni, 2008, p. 159). Dix ans plus tard, c'est encore avec l'UNSA qu'une fusion est désormais envisagée. Le projet d'alliance avec un syndicat actuellement non représentatif mais au poids grandissant permet, dans l'esprit de ses prome teurs, d'allier les militants nombreux de l'UNSA dans la fonction publique avec les forces concentrées dans le privé de la CFECGC, et de transformer cette dernière en un syndicat généraliste consti tuant «une troisième force syndicale». Officialisé au printemps 2008, au lendem ain ou presque de la position commune sur la représentativité, ce projet de fusion et de mue de deux or ganisations complémentaires permet aussi, pour la CFE -CGC, de ne plus dépendre du parapluie, susceptible de devenir guillotine, de l'exception catégorielle. Au sein de la CFE-CGC, les débats sont cependant intenses et même féroces sur ce projet, porté par la direction confédérale, contre l'avis de certaines fédérations et organisations, et pas seulement celles de la fonction 
publique (16). Des fuites dans la presse et un proces sus décidé au sommet sansque la base soit consultée contribuent à crisper les positions. L 'idée d'une fusion glisse alors, face aux oppositions internes, vers l'idée d'une structure chapeau pendant une période transitoire allant de 2009 à 2013, avant qu'un comité directeur, en mars 2009, décide finalement l'abandon du projet et se contente simplement d'affirmer la volonté de créer une «troisième force syndicale à vocation réformiste», pour contester l'hégémonie de la CGT et de la CFDT renforcée par la loi du 20 août 2008.

\section{Un débat permanent aujourd'hui exacerbé : l'identité catégorielle}

Depuis les élections prud'homales de 1997, une «drôle de crise » agite donc la CFE-CGC et touche à son identité. Cette crise aiguise eneffet fondamentalement une interrogation sur l'identité catégorielle de l'organisation syndicale, dont on trouve trace dès les années 1960 et qui avait abouti au changement de nom de 1981 (voir encadré 4).

\section{Encadré 4 \\ Entre cadre et encadrement : une interrogation ancienne}

Dès le début de l'enquête, nous nous sommes heurtés à la question de la population que souhaite représenter la CFE-CGC. Le sigle de la confédération lui-même, long et complexe, en est le premier signe. Entre sa création en 1946 et 1981, la centrale catégorielle représente en effet officiellement «les cadres ", même si l'extension est dès le début maximale. Comme le souligne Paul Meunier (1970, cité par BolTANSKI, 1982), «la CGC accepte tout le personnel d'encadrement sans tenir compte du coefficient hiérarchique. Tout salarié qui détient "une parcelle d'autorité" peut adhérer à la CGC. Ainsi une bonne part des effectifs vient-elle du moyen (agents de maîtrise) et du petit encadrement (contremaîtres) ". Dès les années 1950, se présentent et sont élus des responsables CGC dans le 2e et parfois le 1 er collège, même si ce n'est qu'en 1981 qu'est ajouté le nom de "confédération française de l'encadrement " à celui de "confédération générale des cadres".

Cet élargissement historique témoigne d'une ouverture, mais aussi d'une incertitude qui perdure aujourd'hui et se repère dans les usages variés que font les militants du sigle lui-même. Les uns parlent de la CGC, des cadres; d'autres n'oublient jamais de parler des cadres et de l'encadrement, voire de

(16) En effet, dans la police en particulier, où Alliance, qui appartient à la CFE-CGC, constitue la seconde force militante (et un syndicat au poids important dans la fédération CFE-CGC de la fonction publique), il s'agissait de fusionner avec les concurrents directs, l'UNSA Police constituant à l'époque le premier syndicat. De fait, cette dernière or ganisation ne résistera pas à ce projet puisqu'une majorité de ses membres rejoindra FO, pour fonder Unité SGP Police, seule une minorité restant à l'UNSA Police. l'encadrement uniquement, et prennent soin de corriger systématiquement l'oubli du «CFE». Les renégociations de conventions collectives dans les années 1970, comme au moment des trente-cinq heures au tournant des années 2000 , ont constitué des moments d'interrogations, et en général d'extension du périmètre des salariés que souhaitait représenter l'organisation. Dans la banque par exemple, la suppression du $1^{\mathrm{er}}$ collège et l'organisation en deux collèges cadres et non cadres en 2000 n'ont en rien fait refluer la CFE-CGC sur le seul collège cadre, bien au contraire. Cette évolution fait suite à une importante lutte qui s'est déroulée devant les tribunaux, les autres syndicats contestant systématiquement pendant les années 1980-1990 la possibilité pour la CFE-CGC de présenter des candidats dans les $1^{\text {er }}$ et $2^{\mathrm{e}}$ collèges. Les sections syndicales d'entreprise ont donc dû prouver leur existence et leur représentativité chez les noncadres lors de combats juridiques, incertains et coûteux, même s'ils sont devenus de plus en plus régulièrement victorieux. Prouver sa représentativité - bien avant la loi de 2008 - et définir qui sont les salariés que la confédération souhaite représenter sont ainsi l'objet d'interrogations, de réflexions et de pratiques anciennes au sein de la CFE-CGC.

Les «Assises de la modernité» que la CFE-CGC organise en octobre 1998 avaient manifesté la volonté d'élargir son public à l'ensemble des «professionnels de l'entreprise», dans un ef fort toujours renouvelé d'extension des salariés qu'elle souhaite représenter, au-delà même de ceux « détenant une parcelle d'autorité », pour y inclure tous ceux qui se pensent comme des responsables et des professionnels de l'entreprise : «ce projet n'en reste pas moins vague. Il ne s'agit pas en ef fet de développer une stratégie interprofessionnelle mais de dépasser un carcan statutaire, qui s'érode, tout en demeurant fidèle à un état d'esprit : "être cadre [...] c'est désormais davantage une attitude, une volonté, un projet" déclare la CGC qui croit accomplir ainsi une "véritable révolution"》 (CHOFfat, Verrier, 2006, p. 75).

Tous ces enje ux sont encore présents lors du congrès de février 2010, moins d'un an après la crise au sein du comité directeur à propos du projet de fusion avec l'UNSA. Après plusieurs années de tensions exacerbées ${ }^{(17)}$, le congrès donne finalement l'image d'une confédération qui s'interroge sur sa

(17) En 2005, le décès du président de la CFE-CGC, Jean-Luc Cazettes, élu en 1999, conduit à une sévère querelle de succession au sein de l'appareil. Bernard Van Craeynest est nommé président en décembre 2005, contre Danièle Karniewicz (diri geante de la chimie). Lors du congrès de décembre 2006, il n'est finalement élu qu'avec $66 \%$ des voix. «En 2006, le 33e congrès de la CGC témoigne de tensions internes persistantes, de la nécessité d'une réforme des structures très éclatées pour développer les implantations, de la relance de thèmes tradi tionnels en attendant le renouveau » (CHOFFAT, VerRIER, 2006, p. 76). 
stratégie (voire qui est clivée autour de sa définition), mais qui reconduit sa direction confédérale sans modifier fondamentalement ses orientations. En dépit des débats féroces qu'a suscités la tenta tive de rapprochement avec l'UNSA en 2008-2009, le congrès de Reims se distingue par l'absence d'oppositions déclarées à la ligne proposée par la direction confédérale parti ellement renouvelée. Alors que Bernard Van Craeynest, seul candidat, est reconduit à la tête du syndicat par, cette fois, près de $80 \%$ des votants, Carole Couvert, jeune femme de trente-sept ans issue d'EDF-GDF et tenante de la stratégie visant à construire «une troisième force syndicale» via l'union avec d'autres syndicats, fait une entrée remarquée en position de numéro deux de la centrale. Divisés, les congressistes votent néanmoins massivement pour la direction confé dérale qui se renouvelle donc partiellement. Le caractère relativement policé des débats et le renouvellement sans contestation frontale de la direction confédérale traduisent peut-être un certain épuisement des réflexions, une lassitude des militants face à des enjeux qui s'inscrivent dans une temporalité déjà longue.

\section{Clivages organisationnels et distorsions cognitives dans la représentation}

Pourtant, les hésitations stratégiques que nous venons de décrire dissimulent une polarisation, semble-t-il grandissante, des débats internes, avec une incapacité à dégager une majorité et une minorité au sein du comité directeur de la confédération. Cette polarisation est le résultat de distorsions croissantes dans le travail de représentation du groupe cadre, les dirigeants des vingt -six fédérations et syndicats non fédérés qui composent la CFE-CGC témoignant d'interprétations divergentes de la situation. Autrement dit, si les transformations sociodémographiques du groupe cadre, même plus complexes qu'une simple banalisation, ne sont sans doute pas sans lien avec cette "drôle de crise», leur influence s'exerce par la médiation de l'organisation syndicale et de ses structures. De ce point de vue, la forte homogénéité sociodémographique du groupe des responsables syndicaux de l'or ganisation n'est sans doute pas pour rien dans la distorsion cognitive qui se crée et fait de la CFE-CGC une organisation qui ne représente qu'une partie des cadres et de l'encadrement. Il existe pour autant de forts clivages internes entre les congressistes dans leurs positions, en particulier vis-à-vis du maintien ou non d'une identité catégorielle de leur or ganisation, qui ont partie liée avec les évolutions contrastées des frontières entre cadres et non-cadres dans les différents secteurs d'activité dans lesquels la CFE-CGC puise le gros de ses troupes.

\section{L'homogénéité sociodémographique singulière des congressistes CFE-CGC}

Le congrès de Reims était le premier congrès confédéral auquel assistait la moitié des répondants $(51 \%)$ à notre questionnair e. Si cette proportion peut sembler élevée de prime abord, elle est bien inférieure à ce qui a été observé dans deux autres organisations pour lesquelles nous connaissons ces données : Solidaires (62,2\% de primo-participants en 2008) et la CGT (79,1\% en 2003) (B ÉrOUd, Denis, Desage, Thibaut, 2011 ; Béroud, Garibay, 2004). De fait, un peu plus du quart des congressistes avait déjà participé à plusieurs congrès confédé raux, ce qui marque une ancienneté assez forte dans des fonctions de responsabilité au sein des oganisations qui composent la confédération CFE-CGC et ne dénote pas un renouvellement important au sein d'un groupe socioprofessionnel - celui des cadres qui s'est pourtant rajeuni au cours des années 1990 et 2000 .

De même, et là aussi avec une ampleur signi ficative, l'ancienneté moyenne d'adhésion des congressistes est de vingt ans, $80 \%$ des militants présents à Reims adhérant à l'or ganisation depuis plus de dix ans. Elle reste toutefois bien moins marquée qu'au sein de la CFDT Cadres étudiée par Guillaume et Pochic (2009) : $71 \%$ des délégués cadres au congrès de la CFDTen 2002 avaient adhéré avant 1984 , alors que $41 \%$ des congressistes de la CFE-CGC en 2010 ont adhéré depuis plus de vingt ans - signe d'une possibilité sans doute plus facile de prise de responsabilités au sein de la CFE-CGC qu'au sein de la CFDT. Il est alors logique que les participants au congrès se distinguent des adhérents par une forte surreprésentation des plus âgés au détriment des plus jeunes ( -20 points pour les moins de 40 ans). L'âge moyen des participants au congrès est ainsi de 55,3 ans. Plus que la surreprésentation attendue (les responsables syndicaux sont plus expérimentés et, logiquement, plus anciens et plus âgés que les adhérents), c'est son ampleur qui interpelle : +23 points pour la tranche des 60 ans et plus. La moyenne d'âge est ainsi nettement plus élevée que celle observée lors de congrès confédéraux d'autres organisations syndicales (entre 48 et moins de 50 ans pour FO, la CGT et Solidaires; voir respectivement YON, 2008; BÉROUD, GARIBAY, 2004; Béroud et al., 2011). Surtout, elle va de pair avec une proportion importante de retraités (un congressiste sur cinq), là aussi sans doute plus élevée que dans d'autres syndicats. Alors qu'ils représentent $14 \%$ des adhérents à la CFE -CGC, ils étaient en effet $20 \%$ parmi les participants au congrès ayant répondu au questionnaire.

À cette distorsion générationnelle attendue mais dont l'ampleur paraît frappante quand on la rapporte au rajeunissement du groupe cadre depuis vingt ans, s'ajoute une distorsion également très prononcée en matière de genre. Les chiffres recueillis par Rachel 
SiLVERA montrent que la CFE-CGC est la centrale syndicale française la moins féminisée: en 2002, les femmes y représentaient $18,5 \%$ des adhérents tandis qu'elles étaient $28 \%$ à la CGT, $40 \%$ à FO, $44 \%$ à la CFDT et $46 \%$ à la CFTC (Silvera, 2006) ${ }^{(18)}$. On peut rappeler en outre «la féminisation particulièrement rapide des cadres (l'écart avec les non-cadres [ayant] diminué de moitié en vingt ans)» soulignée par Amossé $(2011$, p. 33), qui indique également " qu'au sein des cadres, on ne comptait que $24 \%$ de femmes en 1982, contre $36 \%$ en 2002, les proportions étant de $43 \%$ et $49 \%$ pour les non-cadres ». En 2008, d'après les chif fres communiqués par la confédération, les adhérentes constituent un quart des effectifs adhérents de la CFE -CGC, soit une augmentation sensible entre 2002 et 2008. Elles représentent néanmoins moins de $15 \%$ des effectifs au congrès de Reims, d'après nos propres données. C'est certes presque le double du congrès de 2002 (elles étaient 8,7\% d'après S ILVERA). Mais à titre de comparaison, le taux de féminisation observé dans les congrès confédéraux d'autres organisations syndicales (FO, la CGT, Solidaires) se situe plutôt entre $25 \%$ et $30 \%$, voire plus. Parmi les cadres présents au congrès de la CFDT de 2002, on comptait même $38 \%$ de femmes (G uillaume, Pochic, 2009).

Autre singularité des congressistes de la CFE-CGC : leur statut de permanent (42\% le sont à temps plein, ce chiffre contrastant avec les indi cations qui nous avaient été données en amont : «il $n$ 'y a pas beaucoup de permanents à la CFE-CGC, même parmi les responsables qui viendront au congrès ») ${ }^{(19)}$ et surtout le type d'entreprise d'où sont issus les délégués. Ces derniers sont ou étaient (pour les retraités), en effet, en grande majorité des salariés du secteur privé ( $80 \%$ des congressistes) et plus particulièrement des grandes entreprises de plus de 500 salariés (pour $86 \%$ de ceux venant du privé). Cela tranche quelque peu avec les congressistes CFDT : seuls $15 \%$ des cadres présents au congrès CFDT de 2002 venaient de grandes entreprises privées, tandis que $20 \%$ provenaient de PME, $14 \%$ d'entreprises publiques et $46 \%$ des trois fonctions publiques (Guillaume, Pochic, 2009). De fait, si des déséquilibres existent dans les deux cas quant à la population cadre, ils sont particulièrement frappants dans le cas de la CFE-CGC tant le public constitue un secteur accueillant un grand nombre de cadres.

(18) Les chiffres de FO et de la CFTC sont ceux donnés par les syndicats eux-mêmes.

(19) Du côté de la CFDT, Guillaume et Pochic décrivent que plus d'un cadre sur deux, au congrès de 2002, est permanent. À la CFE-CGC, le chiffre de $42 \%$ est par ailleurs une moyenne, avec les $34 \%$ de permanents congressistes dans la métallurgie, énergie, construction, $49 \%$ dans la finance, la banque et l'assurance et $57 \%$ dans la fonction publique et les entreprises publiques.

\section{Représenter l'encadrement... ou les seuls (et eux-mêmes hétérogènes) cadres du privé?}

Tout comme l'UGICT-CGT et à la différence de la CFDT Cadres, la CFE-CGC ne prétend pas représenter les seuls cadres, mais bien l'encadrement, et bon nombre de ses adhérents ne sont pas cadres. De ce point de vue, la distorsion or ganisationnelle est totale : car, malgré le nom de la confédération, les cadres sont très fortement surreprésentés au congrès de Reims puisque quatre congressistes sur cinq ayant répondu au questionnaire sont ou étaient cadres, contre un sur deux parmi les adhérents $(52 \%)^{(20)}$. Autrement dit, si la CFE-CGC n'est pas un syndicat auquel n'adhèrent que des cadres, ses instances dirigeantes restent largement dominées par des syndicalistes ayant ce statut. Pour autant, les modes d'accès au statut cadre, et sa signification même, constituent des sources d'hétérogénéité importantes à prendre en considération, et qu'ont soulignées les sociologues depuis une quinzaine d'années au regard de nombreuses dimensions morphologiques, culturelles et professionnelles (BOUFFarTigue, Gadéa, Pochic, 2011). Ainsi, en termes de formation, alors que les responsables cadres de la CFDT sont $53 \%$ en 2002 à avoir un bac +3 et plus, ils ne sont à la CFE-CGC que $42 \%$ à posséder un diplôme de ce niveau, huit ans après, alors que le niveau de formation s'est encore élevé dans la population française ${ }^{(21)}$. De manière certainement corrélée et confirmant une intuition déjà exprimée (mais non étayée statistiquement) par BoLTANSKI (1982) selon laquelle la CGC comportait une part importante de cadres promus, on peut constater que, parmi les cadres présents au congrès, plus de la moitié $(58 \%$ des cadres, soit $44 \%$ de l'ensemble des répondants) n'ont pas débuté leur carrière professionnelle avec ce statut. Cette segmentation se double d'ail leurs ici d'ef fets sectoriels clairement repérables, qui prennent leurs racines dans le poids extrême ment variable des fédérations qui composent la CFE-CGC.

Les deux fédérations les plus fortement représentées au congrès de Reims étaient en effet la métallurgie et la banque, d'où est issu plus d'un congressiste sur deux et qui sont aussi les deux fédérations les plus importantes en nombre d'adhérents. Toutefois, par rapport à ce que la métallurgie représente en nombre d'adhérents, elle

(20) Lors de son précédent congrès, en 2006, la CFE -CGC estimait que les deux tiers de ses adhérents étaient cadres ou ingénieurs (Choffat, Verrier, 2006, p. 76). Si l'on se fie à ces chiffres, cela témoigne d'une transformation sociologique rapide de la base de l'or ganisation, qui ne se manifeste pas parmi ses responsables.

(21) A contrario, et même si l'âge élevé des congressistes lui est lié, $18 \%$ des responsables de la CFE -CGC participant au congrès ont le bac, $12 \%$ un CAP-BEP et plus de $14 \%$ aucun diplôme. 
était surreprésentée au congrès, au même titre que la chimie et au détriment de la fonction publique, voire de l'agroalimentaire (voir tableau 3) ${ }^{(22)}$.

Or, si $41 \%$ des délégués chimistes et $37 \%$ des métallurgistes ont débuté comme cadres, c'est le cas de seulement $18 \%$ des congressistes de la fédération banque / finance, beaucoup plus nombreux à avoir entamé leur carrière avec un autre statut, bien que les cadres y soient, en proportion, aussi nombreux parmi les congressistes que dans les autres fédérations.

Tableau 3 : Fédérations représentées au congrès de Reims 2010 et adhérents en 2008

\begin{tabular}{|c|c|c|c|c|}
\hline \multirow[t]{2}{*}{ Fédérations } & \multicolumn{2}{|c|}{$\begin{array}{c}\text { Congrès Reims } \\
2010\end{array}$} & \multicolumn{2}{|c|}{ Adhérents } \\
\hline & $\begin{array}{c}\% \text { des } \\
\text { participants }\end{array}$ & rang & $\begin{array}{c}\% \\
\text { d'adhérents }\end{array}$ & rang \\
\hline Métallurgie & 33,8 & 1 & 25 & 1 \\
\hline $\begin{array}{l}\text { Finance et Banque } \\
\text { (FFB) (dont Banque de } \\
\text { France et FNECA pour } \\
\text { les congressistes) }\end{array}$ & 18,5 & 2 & 17,2 & 2 \\
\hline Chimie & 9,4 & 3 & 6,1 & 5 \\
\hline $\begin{array}{l}\text { Santé, médecine } \\
\text { et action sociale } \\
\text { (FFASS) }\end{array}$ & 5,5 & 4 & 5,2 & 6 \\
\hline $\begin{array}{l}\text { Union fédérale des } \\
\text { cadres des fonctions } \\
\text { publiques (UFCFP) }\end{array}$ & 5,2 & 5 & 10,1 & 3 \\
\hline Assurance & 4,9 & 6 & $?$ & $?$ \\
\hline $\begin{array}{l}\text { Encadrement du } \\
\text { commerce et des } \\
\text { services (FNECS) }\end{array}$ & 3,9 & 7 & $?$ & $?$ \\
\hline Agroalimentaire & 3,6 & 8 & 6,3 & 4 \\
\hline Autres & 15,3 & - & - & - \\
\hline
\end{tabular}

Données :

- Congrès CFE-CGC Reims 2010; Enquête IDHE; $\mathrm{n}=310$.

- Fichier adhérents CFE-CGC 2008; données fournies par la confédération; $\mathrm{n}=112947$.

Cette sociographie des délég ués au congrès de Reims fait donc apparaître une forte homogénéité sociologique des congressistes qui reflète de plus en plus mal, tant les évolutions morphologiques du groupe des encadrants (féminisation, rajeunissement) que le maintien de leurs clivages, entre cadres et non -cadres (Amossé, 2011), voire leur accroissement (entre public et privé). Cette homogénéité recouvre pourtant de réelles segmentations qui contribuent à distinguer parmi les congressistes de la CFE-CGC, et de manières qui semblent faire système, différentes fédérations (métallurgie vs

(22) Ces rapports des forces entre fédérations ne traduisent qu'imparfaitement les résultats de la CFE -CGC aux élections aux comités d'entreprise dans les divers secteurs d'activité. Les meilleurs résultats sont en effet obtenus dans le secteur des activités financières $(17,2 \%$ des voix, tous collèges confondus en 2005-2006), suivi par les industries des biens d'équipement (dont la construction aéronautique) $(12,2 \%)$, l'énergie (hors EDF-GDF; 10,2\%), les industries des biens intermédiaires (dont la chimie ; 6,9\%), la moyenne nationale s'établissant à $6,5 \%$ (JACOD, 2008b). banque), cadres diplômés et non diplômés, cadres promus et cadres dès l'entrée dans l'emploi, et bien sûr cadres et non-cadres. Elle soulève la question des effets de distorsion dans la représentation que les dirigeants de la CFE-CGC se font de ces évolutions générales.

\section{Attachement et détachement vis-à-vis du syndicalisme catégoriel : fracture organisationnelle et cadrages dissonants}

Invités par le questionnaire à formuler un avis sur les enjeux stratégiques de la confédération, les congressistes ont exprimé les positions les plus clivées sur la question de l'identité catégorielle de leur organisation. L'opposition entre défenseurs du maintien du catégoriel et promoteurs d'une mue généraliste surdétermine les discussions actuelles sur l'avenir de l'organisation. Derrière cette opposition, un «effet fédération » structure très clairement les positionnements respectifs : $76 \%$ des délégués de la métallurgie et $93 \%$ (27 sur 29) de ceux de la chimie choisissent ainsi au moins l'une des trois modalités marquant un attac hement au catégoriel (voir tableau 4), contre $65 \%$ de l'ensemble des participants au congrès. À l'inverse, les délégués des fédérations des banques et des assurances sont plus nombreux à retenir des modalités invitant à se détacher de cette dimension $(66,7 \%$ contre $26 \%$ dans la métal lurgie et un seul individu dans la chimie).

Ces positions clairement opposées reflètent plus largement, derrière une commune défense des salariés d'encadrement, des clivages croissants dans la définition du groupe représenté ainsi que du syndicalisme et de son or ganisation, entre les principales fédérations qui constituent les bastions de la CFE-CGC (23). Dans ces secteurs en effet, les frontières entre cadres et non-cadres ont connu des évolutions contraires. S'appuyant sur une analyse statistique des enquêtes Emploi de l'Insee, Amossé (2011) montre ainsi que la frontière cadres/non cadres est beaucoup plus nette dans l'industrie que dans les services et le commerce. Olivier Cousin (2008) aboutit à une conclusion similaire à l'issue d'une immersion de longue durée dans une grande entreprise métallur gique de haute technologie. Alors que la métallurgie se distingue encore aujourd'hui par l'existence d'une conven tion collective nationale qui couvre exclusivement les ingénieurs et cadres, ce sont des conventions collectives régionales qui couvrent les autres catégories de salariés.

(23) Ensemble, les fédérations finance / banque et assurance (environ $25 \%$ des congressistes), métallur gie et chimie (environ $43 \%$ ) représentent plus de la moitié des adhérents et plus des deux tiers des délégués au congrès de 2010. 
Tableau 4 : Fédération d'appartenance et attachement au catégoriel

\begin{tabular}{|c|c|c|c|}
\hline $\begin{array}{c}\text { Fédération } \\
\text { d'apparte- } \\
\text { nance }\end{array}$ & $\begin{array}{c}\text { Effectif } \\
\text { total des } \\
\text { fédérations }\end{array}$ & $\begin{array}{c}\text { Répondants } \\
\text { citant au moins } \\
\text { une modalité } \\
\text { affirmant l'atta- } \\
\text { chement à la } \\
\text { spécificité caté- } \\
\text { gorielle (*) } \\
\text { Effectif } \\
\%\end{array}$ & $\begin{array}{c}\text { Répondants } \\
\text { citant au moins } \\
\text { une modalité } \\
\text { minorant l'im- } \\
\text { portance de la } \\
\text { spécificité caté-- } \\
\text { gorielle (*) } \\
\text { Effectif } \\
\%\end{array}$ \\
\hline $\begin{array}{l}\text { Banque et } \\
\text { assurance }\end{array}$ & 72 & $\begin{array}{c}\mathbf{2 6} \\
(36,1)\end{array}$ & $\begin{array}{c}\mathbf{4 8} \\
(66,7)\end{array}$ \\
\hline Métallurgie & 104 & $\begin{array}{c}79 \\
(76)\end{array}$ & $\begin{array}{c}\mathbf{2 7} \\
(26)\end{array}$ \\
\hline Chimie & $29 * *$ & $\begin{array}{c}27 \\
(93,1)\end{array}$ & $\begin{array}{c}1 \\
(3,4)\end{array}$ \\
\hline Autres & 105 & $\begin{array}{c}70 \\
(66,7) \\
\end{array}$ & $\begin{array}{c}38 \\
(36,2)\end{array}$ \\
\hline Ensemble & 310 & $\begin{array}{c}\mathbf{2 0 2} \\
(65,2)\end{array}$ & $\begin{array}{c}114 \\
(36,8)\end{array}$ \\
\hline
\end{tabular}

(*) Ont été considérés comme affirmant un attachement à la spécificité catégorielle les répondants citant au moins l'une des trois modalités suivantes à la question 53 («A vos yeux, la dimension catégorielle spécifique à la CFE-CGC est...») : «une dimension qui reste essentielle pour son identité », «une dimension qui reste essentielle pour sa stratégie et son positionnement», «une dimension plus importante encore aujourd'hui»"

Les modalités suivantes ont été considérées comme minorant la spécificité du catégoriel : «une dimension qui n'a jamais été importante», «une dimension historique importante mais qui est un handicap aujourd'hui».

Lecture de la première ligne (Banque et assurance) : dans les fédérations Banques et assurances $(n=72), 26$ congressistes, soit $36 \%$ des répondants de ces fédérations, citent au moins une modalité favorable à la dimension catégorielle à la question $53 ; 48$ individus des mêmes fédérations (soit environ $67 \%$ des répondants de ces fédérations) citent au moins une modalité défavorable à la dimension catégorielle.

** Bien que l'effectif de délégués de la chimie soit le plus faible parmi les grandes fédérations, il nous semble important de présenter les résultats des congressistes de cette fédération de manière isolée tant ils se démarquent des autres par de nombreux aspects. Les autres fédérations, présentant des effectifs trop réduits pour une interprétation rigoureuse de ces résultats, ont été regroupées dans la catégorie «Autres».

Données : Congrès CFE-CGC Reims 2010; $\mathrm{n}=310$

À l'inverse, dans d'autres secteurs et au premier chef les banques ou les assurances, le statut de cadre apparaît bien plus banalisé tant le «contrat de confiance» qui fondait l'appartenance à un groupe distinct s'est rompu et tant s'est renforcée l'unité (symbolique et matérielle) avec le monde salarié. Les propos d'un responsable de la fédération de la banque, liant le moindre attachement actuel des «gens de la banque » au caté goriel à l'affaiblissement général du statut cadre fortement ressenti par les salariés de son secteur, peuvent ainsi être mis en regard de ceux de responsables de la métallur gie, qui, lorsqu' ils n'insistent pas sur la distance qui sépare toujours « le monde de l'ouvrier du monde de l'encadrement», soulignent la multiplicité des cadres aujourd'hui et la difficulté de les représenter, tout en défendant implicitement une distinction toujours pertinente entre cadres et non-cadres.

"Aujourd'hui quoi qu'on en dise, un cadre n'est plus ce qu'il était dans les années 1970. On comprend parfaitement ce qu'a été la CGC à un moment donné, la CFE à un moment donné, parce que le cadre, c'était la charnière indispensable, celui qui faisait le lien entre le patron-je dis bien le patron et pas le dirigeant ou l'actionnaire - et puis la production, l'exécution. $C$ 'était vraiment celui qui recevait les ordres et qui après mettait en application, celui qui pouvait avec le patron essayer de faire modifier telle ou telle chose. Aujourd'hui, les gens ne ressentent plus le statut de cadre comme ça. En tout cas dans le secteur bancaire, c'est clair, un cadre n'est plus vu comme un maillon essentiel de la chaîne de fonctionnement. Il se considère comme à l'interface entre un dirigeant et les actionnaires qui fixent des objectifs et un niveau de rentabilité à atteindre et puis il essaie de mettre ça en adéquation avec les moyens qu'il a sur le terrain. Il n'a plus aucune autonomie en termes d'objectifs, de recrutement. Il est toujours cadre en termes de reconnaissance sociale, mais il n'est plus cadre dans son activité. [...] Tout cela fait qu'on a une population cadre qui avant de se sentir cadre se sent salariée de l'entreprise et puis dans la même galère que tout le monde, avec peut-être un niveau de responsabilité et un niveau de rémunération un peu plus élevés, mais qui n'en font plus des gens à part dans l'entreprise» (un responsable CFE-CGC de la banque).

À l'inverse, un responsable syndical de l'aéro nautique soutient :

«Notre approche à nous, CGC, qui est particulière puisque l'on s'occupe de l'encadrement, c'est de rappeler, c'est comme cela qu'on le conçoit, que le monde de l'ouvrier et le monde de l'encadrement, ce sont des mondes complémentaires et qui n'ont pas les mêmes besoins. Un ouvrier a besoin d'avoir des douches pour se nettoyer le soir pour sortir, etc. Et lorsqu'il rentre chez lui, il n'a pas tiré son enclume avec lui. Le cadre, il arrive chez lui, il a encore tous les problèmes dans la tête: "Qu'est-ce que je fais demain?" Le système de vie est différent et les besoins sont différents donc c'est comme cela que l'on arrive à expliquer qu'on est un syndicat qui s'occupe de l'encadrement» (un élu CFE-CGC d'un grand groupe aéronautique)

... tandis qu'un responsable régional de la métallurgie souligne :

«Mais pour moi, autre chose existe de beaucoup plus fort, qui entache un peu l'aspect catégoriel; c'est qu'un cadre, aujourd'hui, cela ne veut plus rien dire. [...] Car le cadre, aujourd'hui, est multiple. Pour moi, un cadre encadre. Or, de nombreux cadres dans l'entreprise n'encadrent pas, $n$ 'ont pas d'équipe, sont tout seuls. Cela est un peu nouveau. Il n'empêche que nous essayons de les représenter aussi, mais c'est une position différente. Cen'est pas un chef d'équipe, l'élite de l'entreprise comme nous aurions pu le penser avant»" (un responsable régional de la métallurgie).

De fait, comme le souligne une étude récente du Céreq (Möвus et al., 2011), les différences entre secteurs d'activité en matière d'accès à la catégo rie cadres par la voie promoti onnelle restent fortes, le cas typique du secteur financier étant qualifié de modèle de la «voie promotionnelle instituée». Dans ce secteur en effet, plus d'un quart des cadres est issu 
de PCS inférieures, contre $10 \%$ dans la métallurgie et guère plus de $5 \%$ dans l'industrie des biens intermédiaires (qui inclut la chimie). Dans le même sens, dans le secteur financier, plus de $40 \%$ des cadres sont issus de la promotion interne, alors qu'ils sont moins de $25 \%$ dans la métallurgie et la chimie dans ce cas. Enfin, alors que la part des cadres diplômés du $3^{\mathrm{e}}$ cycle et des grandes écoles est de près de $60 \%$ dans l'industrie des biens d'équipement, elle est de moins de $40 \%$ dans le secteur financier.

La «drôle de crise » de la CFE -CGC et, suite à la loi du 20 août 2008, sa cristallisation autour de l'enjeu caté goriel et de la fusion avortée avec l'UNSA révèlent finalement une polarisation croissante de l'organisation autour de quelques bastions qui se fondent sur des professions dont l'évolution sociodémographique est profondément divergente. Cette évolution des structures syndicales alimente une non-congruence des "définitions de la situation » et une divergence dans la manière d'envisager le devenir de la CFE -CGC, au niveau confédéral comme au niveau des sections d'entreprise.

\section{Des perceptions aux pratiques syndicales : syndicalisme d'entre- prise et pléistocratie}

Si la CFE-CGC peine à dépasser ses divisions internes et ses distorsions dans la perception des évolutions sociodémographiques, c'est qu'elle se caractérise d'abord (et peut -être plus que les autres organisations syndicales françaises) par des pratiques syndicales qui sont principalement centrées sur l'entreprise et sur la section syndicale d'entreprise. Cette centration reflète pour partie le rapport que les cadres entre tiennent à l'égard du syndicalisme, et permet d'expliquer que des dissonances cognitives internes puissent provoquer de telles hésitations stratégiques.

\section{Une continuité entre fonction d'encadre- ment et activité syndicale...}

Les études sur l'engagement syndical des cadres soulignent la continuité entre leur pratique syndi cale, le sens donné à leur travail et l'engagement du salarié au service de l'entreprise (BNSOUSSAN, 2010). Si l'engagement syndical des militants de la CFDT Cadres fait, dans certains cas, suite à l'expérience de difficultés professionnelles, «un certain nombre de militants cadres [...] justifient leur engagement par le souhait de participer à la vie collective de l'entreprise. [...] Si, pour l'ensemble des militants, l'engagement syndical est une manière d'être acteur de son environnement de travail, cette dimension prend une tonalité encore plus forte chez les cadres du privé. Ces derniers tissent en effet clairement une cohérence entre leur responsabilité profession nelle à l'égard de leur entre prise - le souci de sa performance et de sa survie - et leur investissement syndical» (Guillaume, Pochic, 2009, pp. 550-551). Cette continuité entre responsabilités profession nelle et syndicale est égalem ent soulignée par les militants de la CFE-CGC. L'expression «participer à la vie de l'entreprise» revient ainsi fréquemment dans leur discours. Elle se traduit par une pratique syndicale fondée sur une légitimité technique ou experte qui se différencie des pratiques militantes traditionnelles. La mobilisation de savoirs experts s'exprime tant au niveau des structures nationales, à travers un " militantisme de dossier» (Delmas, 2009), qu'au sein des entreprises, avec la volonté d'intervenir dans les orientations gestionnaires et techniques des entreprises pour contrebalancer une logique de gestion strictement financière. Ce souci de "continuer à être cadre "d'une autre façon" " (Guillaume, Pochic, 2009, p. 564) alimente un syndicalisme de propositions manifestant la volonté de peser collectivement sur les choix économiques et sociaux de l'employeur. Dans l'aéronautique en particulier, plusieurs interviewés ont mis en avant une volonté de participer à la stratégie industrielle même de l'entreprise :

«Moi, j'essaie d'être constructif, toujours, mais également critique. Mais la critique, pour qu'elle soit constructive, et ça, c'est une spécificité qu'on essaie de maintenir à la CFE-CGC et qui fait que l'on a de bons résultats électoraux, c'est qu'on essaie de ne pas se situer dans le champ uniquement social. Typiquement, le brancardier social. [...] Nous, on essaie d'être en amont, sur le terrain industriel, parce que finalement, $c$ 'est beaucoup les orientations et les choix industriels qui sont faits, de différentes natures, qui conditionnent les problèmes sociaux qu'on aura derrière. [...] On fait aussi des propositions sur les schémas industriels présentés justement pour éviter qu'il y ait des aspects sociaux» (un délégué syndical central CFE-CGC d'un groupe de l'aéronautique).

Les militants affirment une attitude à la fois experte et pragmatique, qui s'appuie sur le niveau de qualification de leurs adhérents et sur le souci de ne pas être perçus comme des «incompétents» et d'être «pris en considération» par les directions d'entreprise. Si le syndicat témoigne d'une respon sabilité dans les échanges avec la direction, qui le conduit à tenir compte descontraintes économiques, il s'attend en retour à pouvoir s'exprimer sur les choix économiques, financiers et stratégiques de l'entreprise.

On touche ici à une constante forte de l'engage ment des cadres dans le syndicalisme. La posture de «force de propositions» et de «syndicalisme responsable» qu'ils défendent fait écho à leur position dans l'entreprise. Car, plus lar gement, le «sens des responsabilités », le «pragmatisme» et la «force de proposition» sont parmi les valeurs les plus fréquemment citées par les congressistes dans les questionnaires. 


\section{... qui se traduit par un syndicalisme centré sur la section d'entreprise...}

Cette posture «responsable» manifeste en même temps un rapport spécifique des militants syndicaux à leur entreprise, qui n'est pas sans reproduire l'attachement qu'entretiennent de nombreux cadres vis-à-vis de leur entreprise et de sondevenir, comme en témoignent nos entretiens.Ils mobilisent, conjointement et parfois alternativement, deux figures de référence : la catégorie "ingénieurs, cadres et encadrement» dans l'ensemble de la société française d'une part, l'ensemble des salariés de l'entreprise d'autre part. Ces deux supports d'identification sont au fondement d'une conception singulière du syndicalisme que l'on peut résumer par la formule selon laquelle le syndicalisme doit viser conjointement les intérêts des salariés et ceux de l'entreprise. Cette représentation n'est pas éloignée de la théorie institutionnelle avancée par le juriste Paul D URAND (1947-1950) qui fait de l'entreprise un ensemble organique dont les parties sont inséparables les unes des autres et tirent leur valeu $r$ de leur union. Cette solidarité d'intérêts en fait une communauté organisée et hiérarchisée dont les membres collaborent sous l'autorité naturelle du chef, qui doit assurer le bien commun des membres de l'entreprise. La défense de l'ordre et de la hiérarchie est unleitmotiv chez les militants de la CFE-CGC (24). Le principe hiérarchique dans les relations de travail constitue une valeur profondément ancrée chez ces derniers, qui, lorsqu'ils s'adressent à des non-cadres, entendent assurer des promesses de progression pour eux. Cet attachement au principe hiérarchique, que relevait déjà Jean-Daniel Reynaud en 1975, nous semble toujours au cœur de l'identitésyndicale de la CFE-CGC : l'entreprise apparaît ainsi comme un vecteur d'attachement et un espace hiérarchique dans lequel se déploie une carrière longue, source d'apprentissage et d'expertise.

Cet attachement fort des militants de la CFE-CGC à la vie de leur entreprise ancre leur activité syndicale dans un périmètre relativement circonscrit dans lequel s'expriment des positions et des pratiques spécifiques. Il renforce un syndicalisme structuré autour de la section syndicale, caractéristique qui n'est propre ni au syndicalisme -cadres ni à la CFE-CGC, mais qui nous paraît particulièrement exacerbée dans ces deux cas. Dans son étude sur les pratiques syndicales d'ingénieurs et de cadres

(24) Ces valeurs alimentent les critiques, acerbes et fréquentes, adressées à la CFDT Cadres, dont les militants de la CFE-CGC dénoncent la défense de l'égalitarisme : «Parce que j'ai jamais souscrit à un discours égalitariste, où ça doit être pour tout le monde pareil, du bas en haut de l'échelle, que ce soit la rémunération, les avantages, tout... Donc je n'adhère pas foncièrement aux idées de la CFDT. Et d'ailleurs, je me demande comment ils arrivent à faire le grand écart entre tout en bas et jusqu'aux ingénieurs 》 (un secrétaire de comité d'entreprise CFE -CGC d'une entreprise de la métallurgie). d'une entreprise de haute technologie de la région nantaise, Anne-Sandrine CASTELOT (2004) décrit ainsi des sections syndicales qui « vivent en autarcie, repliées sur l'univers économique, social et politique de l'entreprise» (p. 147) et manifestant la «volonté d'exprimer une pratique syndicale dans l'entreprise, autour de problèmes rencontrés par l'établissement local et en son sein» (p. 148).

Cette participation syndicale à la «vie de l'entreprise» prend parfois des formes originales. Ainsi, dans la banque, un important travail de communication (qui entend explic itement se distinguer de «l'information revendicative» fournie par les tracts) est accompli par la section syndicale pour compléter délibérément l'action de la DRH (information sur une augmentatio $\mathrm{n}$ de capital réservée aux salariés, sur les primes, etc.), voire conseiller les salariés sur les divers « placements» d'intéressement-participation ${ }^{(25)}$. Dans le secteur industriel, un autre exemple réside dans l'action d'un réseau de délégués syndicaux au sein d'un grand groupe pour faciliter le reclassement de près de deux cents cadres dont l'emploi est menacé suite au rachat de leur établissement par un autre groupe. Le réseau a fait circuler les $\mathrm{CV}$ au sein des dif férentes entités du groupe.

Au-delà de ces pratiques singulières, les militants témoignent d'une conception du dialogue social dans laquelle la consultation est perçue comme le mode privilégié d'échange avec les directions. Cette conception reflète à la fois la conviction qu'il faut des «mécanismes de régulation» dans l'entreprise qui permettent l'expression des salariés et assurent un certain équilibre dans les relations sociales, et la volonté de ne pas fairede ces mécanismes des instruments de blocage. L'attachement à la résolution des problèmes, plutôt qu'à leur seule expression, le refus de toute position dogmatique et la recherche du compromis ont été abondamment évoqués dans les entretiens. Cette représentation des relations professionnelles fondée sur le postulat qui fait de l'entreprise une communauté d'intérêts polarise le positionnement des militants à l'égard du conflit et de la négociation. Lorsque nous soumettons des termes du champ lexical des relations professionnelles à l'appréciation des congressistes, 82,6\% des répondants jugent positivement le terme « compromis » (près de $21 \%$ «très » et $61,6 \%$ «plutôt»), alors que la grève est jugée négativement par $81,6 \%$ des répondants ( $68 \%$ «plutôt» et $13,6 \%$ «très »). Sans surprise, on retrouve des positions analogues dans les discours des personnes rencontrées, qui font rapidement part de leur rétice nce de fond à l'égard de la grève, du conflit ou de l'opposition franche

(25) Notons au passage que la forte valorisation de l'intéressement et de l'actionnariat salarié par la CFE-CGC reflète le souhait de participer au développement économique de l'entreprise, d'y prendre des responsabilités. 
aux directions ou à l'entreprise. Parallèlement, les modes d'action orientés vers le dialogue, la négo ciation ou la recherche de compromis sont mis en avant. La politique des « petits pas» est largement préférée à celle du " grand soir», à l'image de l'engagement de cadres dans d'autres or ganisations syndicales, si l'on suit BENSOUSSAN (2010) qui utilise également cette expression.

\section{... qui alimente l'éclatement organisation- nel de la CFE-CGC et ses dissonances cognitives}

Cette volonté de " s'impliquer» dans la vie de l'entreprise témoigne d'un attachement à une forme de corporatisme d'entreprise (là où la CFE -CGC est fortement implantée, au moins), qui là aussi est ancien et alim ente surtout un sentiment d'autonomie des équipes syndicales à l'égard des structures de la CFE-CGC (26). L'engagement syndical dans la vie de son entreprise et de la section d'entreprise n'implique pas une intégration à un collectif plus large. Une part importante des militants rencontrés, notamment dans les grosses sections, a témoigné d'un faible attachement au destin de la confédé ration, tenant un discours qui, sans le souhaiter , n'exclut pas la possibilité de voir la confédération disparaître, ou d'en sortir. Interrogé sur le projet de «troisième force syndicale», ce délégué syndical central d'une entreprise aéronautique l'exprime très ouvertement : «je sais que c'est peut-être pas politiquement correct ce que je vais dire, mais si demain la confédération CFE-CGC disparaît, je n'en ai strictement rien à faire. Tant que la métallurgie CFE-CGC subsistera, le reste, ça peut s'écrouler, ça ne me dérange absolument pas». Ce type de position manifeste en creux les appuis restreints que ces structures, notamment fédérales, trouvent ou prennent auprès de la structure confédérale. Globalement, dans les questionnaires comme dans les entretiens, les militants interrogés mettent peu en avant le fait de s'appuyer sur les autres structures et niveaux. Les dénominations de nombre de syndicats, dont les principaux, reflètent également cette autonomie des structures. Les militants du SNB (Syndicat national de la banque), d'Alliance ou de l'UNAC (Union des navigants de l'aviation civile)

(26) Ce phénomène n'est pas propre à la CFE-CGC et semble entretenu par le système français de relations professionnelles. Il s'observe également pour les syndicats dans lesquels les «représentations de sens commun ou médiatiques» voient des «organisation[s] particulièrement structurée[s] et contrôlée[s], héritière[s] des principes du centralisme», comme le rappelle Françoise Piotet à propos des enquêtes qu'elle a menées et coordonnées sur la syndicalisation à la CGT. Abordant «le rapport des adhérents et des militants à l'organisation», elle fait part de «l'étonnement» qui fut le sien et celui de ses collègues lorsqu'ils se sont trouvés confrontés, comme d'autres, «à "une joyeuse pagaille", à des syndicats farouchement indépendants » et ayant parfois un rapport très lâche aux structures professionnelles et interprofessionnelles. Voir PIOTET, «Introduction à la troisième partie», in Piotet F., 2009, pp. 243-244. font peu apparaître leur appartenance à la CFECGC. Le syndicat national AED (Aéronautique, espace, défense) bénéficie d'une autonomie de gestion extrêmement importante au sein de la fédération de la métallurgie, et notamm ent à l'égard de ses structures territoriales. Si cette autonomie revendiquée et défendue manifeste un attachement fort au syndicalisme de métier, elle peut ailleurs être plus directement encore attachée à un syndicalisme d'entreprise. La structuration des entités syndicales de la banque suggère ainsi l'image de poupées russes : la FFB (Fédération française des banques), c'est essentiellement le SNB, dont une partie importante des forces se trouve dans le SNB -BNP, lui-même organisé autour du SNB-BNP-Paris.

De notre point de vue, cette autonomie des structures syndicales composant la CFE-CGC n'est pas étrangère à seshésitations stratégiques. Elle alimente une forme de «pléistocratie» interne qui, comme le montrait REYNAUD (1973)(27), d'un côté accentue la capacité à poser des problèmes et des questions et de l'autre, af faiblit la capacité à y répondre par des décisions. La décentralis ation particulièrement accentuée des structures syndicales exacerbe la perception des problèmes, alimentant le sentiment général d'une crise de la CFE-CGC, tout en limitant le rôle des structures confédérales.

La loi du 20août 2008, au-delà de son effet attendu de clarification du paysage syndical, accompagne d'ailleurs cette dynamique d'éclatement organisationnel (phénomène également observé par BÉROUD, YoN et al., 2011), avec un effet de renforcement de la légitimité et des ressources des " bastions». Les choix stratégiques opérés par la CFE-CGC, en termes de développement syndical, entretiennent peut-être finalement cette tendance au renforcement et à l'autonomie des sections d'entreprise déjà bien implantées, et les difficultés pour mutualiser les ressources autour des structures territoriales et interprofessionnelles.

$\begin{array}{ll} & * \\ * & \end{array}$

Les dilemmes stratégiques auxquels fait face aujourd'hui la CFE -CGC ne sont pas nouveaux, mais il semble que sa capacité à les trancher s'érode à mesure que s'accentuent les clivages sectoriels du groupe cadre et encadrement. Toutefois cette «drôle de crise $»$ de la CFE-CGC n'est pas simplementassimilable à une adaptation douloureuse à un contexte socio-économique et institu tionnel profondément

(27) Au lendemain de 1968, ReYNAud décrivait, à travers cette notion, la démultiplication des conflits délocalisés, des groupes d'intérêt et des mobilisations collectives, qui aboutit à la dispersion des problèmes, à une aggravation des difficultés de la décision politique et à un pouvoir accru de la base. 
bouleversé. Les hésitations stratégiques témoignent d'une difficulté des dirigeants syndicaux à s'entendre sur la réponse à donner à ces évolutions et problèmes qui s'inscrivent dans des contextes relativement nouveaux. Elles reflètent des perceptions contradictoires des évolutions de ces contextes, au sein de structures (de plus en plus)polarisées autour de «bastions » dont les évolutions socioprofession nelles sont divergentes.

La capacité historique de la CGC à construire une image cohérente et à organiser un ensemble social flou semble dès lors affaiblie. Et ce justement parce que ce travail de représentation, qui passe d'abord par un accord implicite sur la définition du groupe représenté, est de moins en moins homogène, et qu'il emprunte aujourd'hui à des logiques qui peuvent être différentes, voire contradictoires, selon les fédérations et les sections d'entreprise. Les évolutions divergentes des caractéristiques sociodémographiques des bastions professionnels de la CFE-CGC menacent la cohésion de ces représentations syndicales.

Jusqu'à présent, la CFE -CGC témoigne d'une capacité à faire fi de ces tensions organisationnelles, sûrement liée à la centration des militants syndicaux sur les enjeux directem ent liés à leur section d'entreprise. Mais résistera-t-elle aux échéances à venir? Les dissonances cognitives actuelles ne risquent-elles pas de se transformer en diver gences stratégiques insurmontables?

\section{Bibliographie}

Amossé T. (2004), «Mythes et réalité de la syndicalisation en France», Premières synthèses, n 44.2 , octobre.

Amossé T. (2011), «Cadres/non-cadres : une frontière toujours consistante», in Bouffartigue P., Gadéa C., Pochic S. (dir.), Cadres, classes moyennes : vers l'éclatement?, Paris, A. Colin, pp. 32-45.

Amossé T., Delteil V. (2004), «L’identité professionnelle des cadres en question », Travail et Emploi, no 99, pp. 63-79.

Bensoussan M. (2010), L'engagement des cadres. Pratiques collectives et offres de représentation, Paris, L'Harmattan, coll. «Logiques sociales».

Béroud S., Yon K. (coord.), Dressen M., Gantois M., Guillaume C., Kesselman D. (2011), La loi du 20 août 2008 et ses implications sur les pratiques syndicales en entreprise : sociologie des appropriations pratiques d'un nouveau dispositif juridique, Paris, rapport de recherche Dares.

Béroud S., Garibay D. (2004), Enquête sociologique sur les délégués du 47 congrès confédéral de la CGT (24-28 mars 2003), Noisy-le-Grand, étude Ires-CGT.

Béroud S., Denis J.-M., Desage G., Thibaut M. (2011), L'Union syndicale Solidaires : une organisation au miroir de ses militants, Lyon, étude Solidaires-Triangle.

BÉroud S., Yon K. (2012), «Face à la crise, la mobilisation sociale et ses limites. Une analyse des contradictions syndicales », Modern \& Contemporary France, Vol. 20, no 2, pp. 169-183.

Boltanski L. (1982), Les cadres. La formation d'un groupe social, Paris, Minuit.

Bouffartigue P. (2001), « Les métamorphoses d'un salariat de confiance : les cadres dans le tournant des années quatre-vingt-dix», Travail et Emploi, no 86, pp. 107-125.
Bouffartigue P., Gadéa C., Pochic S. (dir .) (2011), Cadres, classes moyennes : vers l'éclatement?, Paris, A. Colin.

Castelot A.-S. (2004), « Le foyer syndical, au cœur d'une pratique syndicale», Cahiers $d u$ GdR Cadres, no 7 , pp. 147-153.

Choffat T., Verrier B. (2006), « Le regain de l'action catégorielle : CGC, G-10-Solidaires, FSU, UNSA...», in Andolfatto D. (dir.), Les syndicats en France, Paris, La Documentation française, pp. 71-95.

Cousin O. (2008), Les cadres à l'épreuve du travail, Rennes, PUR.

Delmas C. (2009), « Un usage militant de l'expertise. Les organisations de cadres face à la souf france au travail», in Crespin R., Bérard Y. (dir.), Aux frontières de l'expertise. Dialogues entre savoirs et pouvoirs, Rennes, PUR, pp. 131-144.

Delmas C. (2011), «"Stress” et "souffrance au travail” des cadres : enjeu syndical et média tique», in Bouffartigue P., Gadéa C., Pochic S. (dir.), Cadres, classes moyennes : vers l'éclatement?, Paris, A. Colin, pp. 219-227.

Denis J.-M. (2005), « Les cadres et l'action collective : un rapport ambivalent», Cahiers $d u$ GdR Cadres, no 10 , pp. 74-84.

Durand P., Jaussaud R. (1947-1950), Traité de droit du travail, Paris, Dalloz.

Frege C.M., K elly J. (2004), Varieties of Unionism: Strategies for Union Revitalization in a Globalizing Economy, Oxford et New York, Oxford University Press.

Giraud B. (2006), «Au-delà du déclin. Difficultés, rationalisation et réinvention $d u$ recours à la grève dans les stratég ies confédérales des syndicats ", Revue française de science politique, Vol. 56, n 6, pp. 943-968. 
Groux G. (1983a), «Les couches moyennes, l'État et le corporatisme en France», Revue française de sociologie, Vol. 24, no 2, pp. 301-315.

Groux G. (1983b), Les cadres, Paris, La Découverte, coll. «Repères ».

Groux G. (1990), « Le syndicalisme-cadres de l'aprèsguerre à nos jours. Légitimation sociale et "affirmation de soi" "), in Reynaud J.-D., Eyraud F., Paradeise C., Saglio J. (dir.), Les systèmes de relations professionnelles. Examen critique d'une théorie, Paris, Éd. du CNRS, pp. 133-145.

Grunberg G., Mouriaux R. (1979), L'univers politique et syndical des cadres, Paris, Presses de la Fondation nationale des sciences politiques.

Guillaume C., Pochic S. (2009), «Un engagement incongru? Les cadres et le syndicalisme, l'exemple de la CFDT», Revue française de science politique, Vol. 59, $\mathrm{n}^{\mathrm{0}} 3$, pp. 535-568.

Hunt S.A., Benford R.D., SnOw D.A. (1994), « Identity fields: framing processes and the social construction of movement identities », in Larana E., Johnston H., Gusfield J.R. (eds), New Social Movements: From Ideology to Identity, Philadelphia: Temple University Press, pp. 185-208.

JACOD O. (2008a), Les élections aux comités d'entreprise de 1989 à 2004 : une étude de l'évolution des implantations et des audiences syndicales, Document d'études Dares, no 137, avril.

JACOD O. (2008b), « Les élections aux comités d'entreprise en 2005-2006», Premières informations, $n^{\circ} 40.3$, octobre.

McAdam D., Tarrow S., Tilly C. (2001), Dynamics of Contention, Cambridge, Cambridge University Press.

Meunier P. (1970), «Le syndicalisme des cadres», Droit social, $\mathrm{n}^{\circ} 11$, pp. 506-516.
Möbus M. (avec Delanoë A. et Ryk F.) (2011), «Devenir cadre par la promotion. Données de cadrage», Notes emploi formation, Céreq, no 47.

Pernot J.-M. (2005), Syndicats : lendemains de crise?, Paris, Gallimard.

Pernot J.-M., Pignoni M.-T. (2008), «Les salariés et les organisations syndicales de 1992 à 2004 : une longue saison de désamour ", in Amossé T., Bloch-London C., Wolff L. (dir.), Les relations sociales en entreprise. Un portrait à partir des enquêtes « Relations professionnelles et négociations d'entreprise», Paris, La Découverte, pp. 140-162.

Piotet F. (dir.) (2009), La CGT et la recomposition syndicale, Paris, PUF, coll. «Le Lien social».

Reynaud J.-D. (1973), « Tout le pouvoir au peuple ou de la polyarchi e à la pléistocratie », in Une nouvelle civilisation? Hommage à Georges Friedmann, Paris, Gallimard, pp. 76-92.

Reynaud J.-D. (1975), Les syndicats en France, t. I, Paris, Le Seuil.

Rouban L. (2001), «Les cadres du privé et du public: des valeurs sociopolitiques en évolution», Revue française d'administration publique, no 98, pp. 329-344.

Silvera R. (2006), «Le défi de l'égalité hommes/femmes dans le syndicalisme», Mouvements, no 43, pp. 23-29.

Turner L., Katz H., Hurd R. (2001), Rekindling the Movement: Labor's Quest for Relevance in the 21st Century, Ithaca et Londres, ILR Press -Cornell University Press.

YoN K. (2008), Retour sur les rapports entre syndicalisme et politique : le cas de la CGT-FO : éléments pour la sociologie d'un "monde de pensée », doctorat de sciences politiques, université Paris-I. 